OPTICAL MEASUREMENTS OF DEGRADATION IN AIRCRAFT BOUNDARY LAYERS*

\author{
Dennis Kelsall \\ Massachusetts Institute of Technology \\ Lincoln Laboratory \\ Lexington, Massachusetts 02173
}

\begin{abstract}
A review is given of visible wavelength measurements of the degradation of optical beams when transmitted through the thin aerodynamic boundary layers around an aircraft.
\end{abstract}

"The views and conclusions contained in this document are those of the contractor and should not be interpreted as necessarily representing the official policies, either expressed or implied, of the United States Government."

*This work was sponsored by the Department of the Air Force. 


\section{OPTICAL MEASUREMENTS OF DEGRADATION IN AIRCRAFT BOUNDARY LAYERS*}

\section{SUMMARY}

The optical degradation produced at the turbulent boundary layer near the skin of a high altitude jet aircraft has been studied in detail over the past several years by M.I.T. Lincoln Laboratory. Some early investigations into the inaging of stars by a telescope located on a $\mathrm{KC}-135$ jet aircraft indicated that the optical degradation of these images might be attributed to an in-flight aircraft boundary layer.

Interferometric techniques to measure these degradations in terms of an optical modulation transfer function (MIF) were developed and a wide range of both wincltunne1 and aircraft field measurements were subsequently conducted by M.I.T. Lincoln Laboratory. The measured MTF data could be curve fitted to an expression for the time averaged MTF of the following form

$$
<\mathrm{D}(\mathrm{s}, \Psi)>=\mathrm{D}_{0}(\mathrm{~s}) \exp -\mathrm{k}^{2} \sigma^{2}\left\{1-\exp -\left(\frac{\mathrm{sD}}{2 l}\right)^{2}\right\}
$$

where $\mathrm{D}_{0}(\mathrm{~s})$ is the optical telescope diffraction 1 imited MTF, with a diameter $D$ and focal length $f, k$ is the wavelength constant, $s$ is the reduced spatial frequency parameter given by $s=2 \lambda R f / D$. Here $\lambda$ is the wavelength in millimeters, and $R$ the spatial frequency in cycles per millimeter in the image plane.

Therefore, from these measurements, the rms wavefront distortion $(\sigma)$ and the correlation length ( $(\ell)$ due to the boundary-1ayer degradation for the transmitted light beam could be determined. These values were characteristics of the aperture diameter $D$, but could be extrapolated to equivalent infinite aperture values $\sigma_{0}$ and $\because 0$ by means of aperture scaling relationships.

Analytical evaluation of the expected $\sigma$ and $\ell$ values from aerodynamic theory has been carried out by $D^{\prime}$ Amato, and some of these theoretically derived values are compared with the experimentally obtained data discussed here.

A summary of the measurements and data obtained in experiments on a Lear Jet Aircraft and on KC-135 airplanes is described here.

\footnotetext{
*'This work was sponsored by the Department of the Air Force.
} 
A turbulent aerodynamic boundary layer near the skin of a high-altitude jet aircraft can degrade the optical quality of a light beam propagated from an aircraft platform. Stine and Winovich ${ }^{1}$ first reported an experiment in which the scattering of a light beam due to a turbulent boundary layer, generated inside a wind tunnel, was measured. From these measurements, both the average integral scale and the intensity of the density fluctuations could be estimated. The results of the Stine and Winovich experiment have subsequently been analyzed by Fufnagel, ${ }^{2}$ Sutton, ${ }^{3}$ Veed and Tuttle ${ }^{4}$ amongst others, in order to estimate the probable optical degradation that can be expected by propagating an optical beam through an aerodynamic boundary layer.

Measurements made from a high-altitude jet aircraft (a KC-135), obtaining Modulation Transfer Function data by photographing star images and analyzing these in the laboratory to evaluate the overall atmospheric degradation (over a long path), have been reported by Luke. ${ }^{5}$ More recently, a series of experiments to identify and measure the various sources of airborne image degradation was carried out by M.I.T. Lincoln Laboratory. The results of photographic experiments were discussed by Bryant, ${ }^{6}$ while the results of interferometric electrooptical experiments were reported by Kelsall. ${ }^{7}$ An analysis of the aerodynamic cffects on airborne optical systems was carried out and outlined by Wolters. ${ }^{8}$ Nevertheless, at this stage in the study of aerodynamic boundary layers, there was a complete lack of any detailed set of experimental data for airplanes over any known and controlled range of flight conditions. It was difficult, if not almost impossible, to predict with any certainty the optical degradation of a light beam propagated out from an aircraft in flight. This was because of the Ilany variables that one must take into account in an aerodynamic environment, as was pointed out by Visinski. ${ }^{9}$ A simple theoretical approach to the problem was found to be inadequate when preliminary attempts to compare this simple theory with experiment were made. $10,11,12$.

There was no readily available instrumentation or method in existence for carrying out these detailed investigations, and for obtaining a large amount of reliable data. It was necessary to explore and develop new optical techniques for carrying out these experiments satisfactorily, 13,14 and it was necessary to define and perfect corresponding data reduction techniques in order to handle and reduce the large amounts of data by computer methods. Finally, these data must be analyzed, first optically, and then correlation of the results made with the physical and aerodynamical properties of the air turbulence regions through which optical beams were propagated. 


\section{OPTICAL THEORY}

A simplified outline of the optical theory will be given in order that the physical-optical processes can be understood and that the approach adopted to carry out the optical analysis of the data can be explained. No account of the aerodynamic analysis will be given here since this is reported by D'Amato. ${ }^{12}$

If a light beam is propagated through a region in which refractive index IJuctuations caused by density fluctuations in the turbulent media are present, the resultant beam is optically degraded. If $f(x, y)$ is the complex amplitude of the degraded beam (in the pupil plane), then the complex amplitude in the image jilane will be $A(u, v)$ where these two functions are Fourier transform pairs

$$
\begin{aligned}
& \mathrm{f}(\mathrm{x}, \mathrm{y}) \stackrel{\rightleftarrows}{ } \mathrm{A}(\mathrm{u}, \mathrm{v}) \\
& \mathrm{I}(\mathrm{u}, \mathrm{v}) \vec{\ddagger}|\mathrm{A}(\mathrm{u}, \mathrm{v})|^{2}
\end{aligned}
$$

and where $(x, y)$ represent coordinates in the pupil plane, $(u, v)$ represent coordinates in the image plane, and $I(u, v)$ is the image intensity distribution (Fig. 1).

The optical transfer function (OTF), D $(s, \psi)$ can be written as follows:

$$
D(s, \psi)=M(s, \psi) e^{i \theta(s, \psi)}
$$

and

$$
D(s, \psi)=\int_{-\infty}^{\infty} I(u, v) \exp [-i(u \cos \psi+v \sin \psi) s] d u d y
$$

where $D(s, \psi)$ is the one-dimensional OTF, $M(s, \psi)$ is the one-dimensional MTF, and $\theta(s, \psi)$ is the one-dimensional phase transfer function (PTF). The OTF, D (s, $\psi$ ), is measured along an optical diameter along a direction which makes an angle $\psi$ with the $x$-axis. The parameter $s$ is a reduced spatial frequency which can be written as

$$
\mathrm{s}=\frac{\lambda \mathrm{R}}{\sin \alpha}=2 \lambda \frac{\mathrm{Rf}}{\mathrm{D}}
$$

where $\alpha$ is the angular aperture size, $R$ is the spatial frequency in the image plane of the optics of diameter $D$ and focal length $f$, and $\lambda$ is the wavelength of 1 ight.

In practice, the OTF can be measured by means of a shearing interferometer designed to take the autocorrelation of the pupil function ${ }^{15,16} \mathrm{f}(\mathrm{x}, \mathrm{y})$ :

$$
D(s, \psi)=\iint_{A} f(x, y) f^{*}(x-s, y) d x d y
$$


where $A$ is the area of the interferogram. Details of the optical Fourier theory which describes these techniques are in Reference 17.

The complex pupil function, $f(x, y)$ describes the amplitude $A(x, y)$ and phase $\psi^{\prime}(x, y)$ conditions over the wave front and is given by

$$
f(x, y)=A(x, y) e^{-i \phi(x, y)}
$$

In those cases where there is uniform irradiation over the cross-sectional area of the beam, $A(x, y)$ does not normally vary significantly and has little effect on the transfer function, even for large variation of $\phi(x, y)$, and for practical purposes we write $A(x, y)=1$.

The phase component, $\phi(x, y)$, is measured from a fixed reference sphere (see Fig. 1), and with respect to this reference sphere can be either positive or negative. If we consider the wave front at one instant of time, the "best fit" to the wave front can be represented by $\Phi(x, y)$ as shown in Fig. 1 . If we write the optical wave front distortion at each $x, y$ point as $\Delta \phi(x, y)$, then

$$
\Delta \phi(x, y)=\overline{\phi(x, y)}-\phi(x, y)
$$

In principle, the fast shearing interferometer (FSI) measures the optical transfer function which depends only on the $\Delta \phi(x, y)$ distribution over the wave front. The mean phase term, $\Phi(x, y)$, does not influence the measurement made by the FSI. This $\overline{\phi(x, y)}$ term will include a tilt component (which again does not influence the interferometer measurement of the OTF). In the case of thin turbulent air regions, such as aircraft boundary layers, the tilt term is expected to be relatively small although it cannot be ignored. In the case of propagation through the random refractive index medium of the atmosphere, it will be more appropriate to take the averaged value of the OTF. It has been shown by $0^{\prime} \mathrm{Neil1}^{18}$ and Barakat ${ }^{19}$ that the averaged value of the optical transfer function can be written in the form ${ }^{\dagger}$

$$
\left\langle D(s, \psi)>=D_{0}(s) \exp -k^{2} \sigma^{2}\left\{1-\exp -\left(\frac{s D}{2 l}\right)^{2}\right\}\right.
$$

where $\mathrm{D}_{\mathrm{O}}(\mathrm{s})$ is the deterministic transfer function of the optics (usually this is diffraction limited) whose receiving diameter is $D$. Here $k$ is the wave number, $\sigma$

\footnotetext{
Here Barakat tentatively assumed a Gaussian correlation function. In I)'Amato's aerodynamic analytical treatment, he found it more appropriate to use an exponential correlation function. This will only introduce a small difference in the resulting correlation length evaluation, of the order of 10 percent difference, and for all practical purposes here need not be of any concern. The Gaussian correlation function yields a correlation length $\ell$ which is smaller by the factor $\sqrt{\pi} / 2$ or 0.89 times the length derived using an exponential correlation length.
} 
is the optical rms wave-front distortion, and $s$ is the reduced spatial frequency (or shear value). The length $\ell$ is a correlation length related to the correlation between the density fluctuations in the medium of the atmosphere. Here $\sigma^{2}$ is the averaged optical mean-square wave-front distortion given by

$$
\left.\sigma^{2}=\overline{\Delta \phi^{2}(x, y)}\right\rangle
$$

where the over bar denotes spatial averaging over the pupil of diameter D, and the brackets $<>$ denote an ensemble average which must be taken because the parameters are varying with time. The main features in the steps to calculate the optica1 rms phase distortion will be outlined here in order to more clearly describe the treatment of the experimental data obtained, and to clarify the relationships between the optical theory and the aerodynamic analysis of D'Amato. ${ }^{12}$ Hogge ${ }^{20}$ discussed a similar approach to the analysis of the Streh1 Ratio. From Eqs. (5) and (7)

$$
\begin{aligned}
\sigma^{2} & =\left\langle\overline{\overline{\phi(x, y)}-\phi(x, y)]^{2}}\right\rangle \\
& =\left\langle\overline{\phi(x, y)}>-\left\langle\overline{\phi(x, y)^{2}}\right\rangle\right. \\
& =\sigma_{0}^{2}-\left(\sigma^{2}+k^{2}\right)
\end{aligned}
$$

the spatial averaging (overlap) being done over the area of the pupil (diameter D) where

$$
\begin{aligned}
\sigma_{0}^{2} & =\left\langle\overline{\phi^{2}(x, y)}\right\rangle \\
\frac{2}{\sigma} & \left.=\left\langle\bar{\phi}^{2}\right\rangle \quad \text { (independent of } x, y\right) \\
\overline{\mathrm{k}^{2}} & =\left\langle\mathrm{k}^{2}\left(\mathrm{x}^{2}+y^{2}\right)\right\rangle \quad(\text { the tilt term) } \\
\overline{\phi(x, y)} & =\bar{\phi}+k(x \cos \phi+y \sin \phi)
\end{aligned}
$$

and $\mathrm{k}$ is the amount of tilt measured along an axis making an angle $\phi$ to the $x$-axis. Equation (8) can also be written in the general form

$$
\left.\sigma^{2}=\sigma_{0}^{2}-\pi \int_{0}^{D} C_{\phi}(s I)\right) \quad D_{0}(s) s d s
$$


where $C_{\phi}(s D)=\left\langle\overline{\left(x_{1}, y_{1}\right) \quad \phi_{\phi}\left(x_{2}, y_{2}\right)}\right\rangle$ for $x, y<D$ is the covariance function (which here includes the mean fluctuation and any components due to tilt of the wave front), both of which can be evaluated from the aerodynamics. $D_{0}(s)$. is the diffraction-1imited optical transfer function (of the receiving optics). In this way, the o value measured optically can be compared with the predictions from theory, computing the corresponding values from aerodynamics. A correlation length $\ell$ can also be computed from the aerodynamics as described by $D^{\prime}$ Amato ${ }^{12}$ and compared with the optically measured values. It should be noted that the optical value of $\sigma$ obtained depends on the diameter of the optics used to make the measurement determined by the second factor in Eq. (8). As $\mathrm{D} \rightarrow \infty$, then $C_{\phi}(S D)=0$ and $\sigma \rightarrow \sigma_{0}$. It is assumed that over the observation times in question that $\langle\phi(x, y)\rangle=0$ and $\langle\overline{\phi(x, y)}\rangle=0$.

In summary, in order to evaluate the optical degradation $\sigma^{2}$ in an optical beam, the different degradation components which make up Eq. (9) must be taken into account and where necessary, scaled with aperture diameter. The result will depend on whether a short averaging time (tilt independent). or a long averaging time (tilt dependent) is considered. In order to calculate the MTF (from which the image intensity pattern can be calculated by a Hankel Transform) or the Strehl Ratio, the optical degradation $\sigma_{S}^{2}$ or $\sigma_{L}^{2}$ is first found as follows:

(1) Tilt-independent (short time average) degradation:

$$
\sigma_{s}^{2}=\sigma^{2}=\sigma_{0}^{2}-\bar{\sigma}^{2}-\overline{\mathrm{k}^{2}}
$$

(2) Ti1t-dependent (long time average) degradation:

$$
\sigma_{L}^{2}=\sigma^{2}+{\overline{k^{2}}}^{2}=\sigma_{o}^{2}-\bar{\sigma}^{2}
$$

The bar refers to spatial averaging over the optics diameter $D$ where $\alpha$ is the optical degradation, $\overline{\mathrm{k}^{2}}$ is the tilt component degradation, $\overline{\mathrm{a}}^{2}$ is the total mean variance, and $\sigma_{0}^{2}$ the total fluctuation yariance of the wave-front distortion. For large (infinite) apertures, $\sigma_{S}=\sigma_{L}=\sigma_{0}$ depends on the density fluctuations in the boundary layer and can be calculated as described by D'Amato from the aerodynamics, while $\sigma^{2}$ and $\overrightarrow{\mathbf{k}^{2}}$ approach zero in this case. From the calculations of D'Amato, the form of $\sigma_{S}^{2}$ and $\sigma_{L}^{2}$ vs $\left(\ell_{o} / D\right)$ is shown in Fig. 2. For small optical 
diameters, the effective optical degradation decreases very significantly, while the effect of the tilt term (shown by the difference between $\sigma_{L}^{2}$ and $\sigma_{\mathrm{s}}^{2}$ ) is relatively small and becomes negligible for large diameters. The largest tilt component for any aperture here will only produce roughly an additional 0.10 amplitude drop in either the MTF degradation or in the Strehl Ratio, and for most practical purposes here can be neglected. This aperture scaling behavior describes the measured variation of $\sigma$ with aperture in the experiments with optics diameters of 25,50 , and $89 \mathrm{~mm}$, respectively, and also accounts for the observed differences between the 89- and 178-mn optics. The Lear Jet data were taken with a 25 -rm optics diameter, and the boundarylayer thickness and the correlation lengths are much less than in the later KC-135 airplane experiments, and these factors are reflected in the data in question.

The scaling relationship for the correlation lengths derived by $D^{\prime}$ Amato are shown in Fig. 3 where $(l / D)$ is shown plotted vs $\left(\ell_{0} / D\right)$. From this plot for a measured $\ell$ (with the FSI), for a given beam diameter $D$, the infinite aperture correlation length $\left(l_{0}\right)$ can be determined, and then scaling to any other beam diameter can be obtained. The corresponding $\ell_{0}$ determined in this manner can then be used by reference to Fig. 2 to scale the measured $\sigma$ for a given diameter to any other beam diameter. The corresponding beam degradation for $\sigma, \ell$, and $\mathrm{D}$ can then be computed by means of Eq. (6) to evaluate the MTF.

There remains one further possible limitation that arises with regard to measuring the time-averaged MTF and using Barakat's Eq. (6), which refers to the averaged OTF, and carrying out the curve-fitting operation to evaluate $\sigma$ and $\ell$. In the experiments described here, the data from the FSI can yield both the MTF (proportional to signal amplitude), and the phase transfer function PTF (determined from the carrier frequency or the signal phase measurement). The platform on which the FSI was located however, was subjected to seyere vibrations and was not spatially stable. These instabilities give rise to angle-ofarrival fluctuations in the optical beam measured by the FSI, producing additional fluctuations in the carrier signal frequencies. They do not however, affect the amplitude of the signal. While accurate MTF data can be obtained in these field environments, it is not possible to process the data and extract the phase transfer function because of the larger, noisier, angle-of-arriyal fluctuations. It requires a very stable environment to obtain accurate phase transfer function data, even in the laboratory. Therefore, it is important to consider the question 
of whether the average MTF data measured here are equa1 to the averaged OTF, and whether one can curve fit our experimentally measured data to an expression of the form given by Barakat. It has been shown by both Cook $^{21}$ and Weaver ${ }^{22}$ that a simple analysis of the problem leads to a relationship of the following form

$$
<D(s, \psi)>=<M(s, \psi)>\text { e } \frac{-i \Delta^{2}}{2}
$$

where $\Delta^{2}$ is the variance of the phase transfer function. In principle, the variance $\Delta^{2}$ is a measure of the amount of nonsymmetry in the fluctuations in the transfer function. It might be expected that in an approximately isotropically random fluctuating medium that $\Delta^{2}$ might be small, and particularly in the case of a thin turbulent boundary layer, it is provisionally assumed that $\Delta^{2} \ll \sigma^{2}$ where $\sigma^{2}$ is the variance of the wave-front distortions. If this is true, then as a first approximation

$$
\exp -\frac{i \Delta^{2}}{2} \rightarrow 1 \text { and }\langle D(s \psi)>\rightarrow\langle M(s \psi)\rangle
$$

In order to test this hypotheses, the measured data were test fitted to the Barakat expression Eq. (6). Good curve fits to the measured data were obtained and it was concluded that the average MTF data measured could be used to evaluate the optical wave-front variance $\sigma^{2}$ as explained earlier. An outline of the theory of operation of the fast scanning interferometer used to measure the MTF is given in Reference 13, and this technique will not be described here.

\section{STAR SOURCE DEGRADATION MEASUREMENTS}

In some early measurements of the imaging of stars taken from a KC-135 aircraft in flight, a degradation of the images was observed, which was attributed to the flight environment. Using a corner cube shearing interferometer and an $89 \mathrm{~mm}$ diameter receiving telescope, MTF measurements were obtained from star source observations with the airplane flying between $7 \mathrm{~km}$ and $12 \mathrm{~km}$ altitude. These data were we11 below the diffraction limited capability of the telescope employed, and some representative data are shown in Fig. 4, for four different star sources viewed (these are averages of several points). In these experiments cach MTF point was recorded on tape, but took up to a minute for a single point, and only a limited amount of data could be obtained. Nevertheless, a boundarylayer degradation was observed, and several series of different experimental configurations were employed to obtain confirmation of the results, as was described in Reference 7. 


\section{LASER SOURCE FAST SHEARING INTERFEROMETER MEASUREMENTS}

A much more detailed and comprehensive set of boundary-layer measurements has subsequently been carried out. For this program a fast shearing and very stable triangular configuration interferometer was designed and built. A more extended series of experiments on airplanes, in which the flight conditions were carefully controlled, has been carried out and will be outlined here.

\section{A. LEAR JET EXPERIMENTS}

In this experiment, the FSI was mounted on a bench in the Lear Jet aircraft and an airfoil mounted outside a window in the airstream to hold a small mirror about $25 \mathrm{~cm}$ away from the skin of the aircraft. An optical schematic shown in Fig. 5 illustrates how the optical degradation in the boundary-layer airstream between this mirror and the aircraft window was examined to obtain MrF data. A 5-mW HeNe CW 1aser was mounted underneath the FSI. A high-quality 50-mm-aperture laser beam expanding telescope was used to provide a col1imated and coherent laser beam. A mirror and a beam splitter were arranged to direct the 50 -mm collimated laser beam through a 75-mm-diameter window in the side of the aircraft. The bean then propagated through the airstrean (the boundary layer) to a 30-mmdiameter gold-coated mirror supported by the airfoil outside the aircraft.

It was reflected back from this mirror again through the boundary layer and then back through the window to the inside of the airplane. Experiments were also performed with an aerodynamically designed tube (an airflow shield) fitted prior to a flight between the gold-coated mirror and the $75-\mathrm{mm}$ optical window to enclose completely and shield the $25-\mathrm{cm}$ light path outside the aircraft from the flight boundary layer.

The returned laser beam finally passed through the beam splitter to a 24-mmaperture telescope which reduced the laser beam in diameter to about $8 \mathrm{~mm}$. This then entered the interferometer. The auxiliary optical components arranged in this experimental setup were of very high optical quality to ensure that the wave-front entering the interferometer in the absence of the boundary layer was plane to within one-tenth wave. A photograph of the Lear Jet airplane is shown in Fig. 6. The airfoil mounted outside the window forward of the wing and near the entry door at point A can just be seen, the optical axis being located $4.9 \mathrm{~m}$ from the airplane nose. The mounting of the FSI, the optics for the autocollimation system, and the laser source located inside the aircraft, are shown in Fig. 7. The electronics are mounted in the rack on the left. The flight conditions could 
be varied over a wide range. At each condition, the effect on the MTF could be observed in real time, focus adjustments (on the receiving-beam reducing optics on the front of the FSI) could be quickly and accurately made, and the alignment of the system could be checked and corrected (since in flight due to aerodynamic loads, the airfoil changed slightly and consequently caused some changing of the optical alignment).

\section{B. $\mathrm{KC}-135$ OPTICAL PROPAGATION EXPERIMENTS}

The optical propagation experiments were directed towards evaluating and characterizing the optical degradation measurements from an aircraft platform. Propagation measurements were made through the local aircraft boundary layers as well as over longer paths (free-stream atmospheric regions). Two similar KC-135 airplanes were instrumented for these experiments. Optical propagation measurements were conducted from each of these two aircrafts. Visible beam propagation measurements (at one wavelength $\lambda=632.8 \mathrm{~nm}$ ) were made through the window just behind the wing in the aft airplane section in the side of the \#1 airplane (located $24.8 \mathrm{~m}$ from the nose of the airplane).

Similar visible beam propagation measurements were made through the forward section window (located $10.4 \mathrm{~m}$ from the nose of the airplane) on aircraft \#2. In practice, the two airplanes were arranged to fly together in formation at a suitable distance apart which varied from about 0.30 to $3 \mathrm{~km}$. In this measurements program it was difficult to always have control of the measurement conditions. Weather conditions intervened, flights sometimes had to be aborted or limited. It was not possible to always optimally perform these field measurements.

A schematic is shown in Fig. 8 to illustrate the series of visible-wavelength measurements through the two airplane windows, one forward on aircraft \#2, the second aft on aircraft \#1. A visible-wavelength laser beam was propagated from one airplane to the other (this could be done in either direction). A second configuration allowed the visible laser beam from the aft window on aircraft \#1 to be directed at a retroreflector located on its wingtip. On reflection, the beam retracted its path and passed through the same window for making measurements. A photograph showing the position of the retroreflector on the wingtip of aircraft \#1 seen from the aft window is shown in Fig. 9 which also shows aircraft \#2 flying in the distance.

The distance from the retroreflector to the optics inside the aircraft was $18.3 \mathrm{~m}$. Note that in this case the outgoing beam passing through the boundary layer was quite small although it irradiated the whole retroreflector (with enough 
overlap to keep it irradiated uniformly as the wing moved up and down during flight). A spherical reflected beam, whose origin was close to the retroreflector on the wingtip, was then propagated back to the aircraft and received by the $89-\mathrm{mm}$ optics. The MTF degradation measurement was therefore representative of one single pass, as the beam made the return trip from the retroreflector through the boundary layer, the beam diameter being determined by the receiving optics aperture.

In the flight experiment using the two aircraft, the optical degradation produced in the boundary layer outside each window was measured using the FSI instrument which could be located on an optical bench on either aircraft just inside the window. It was necessary to use an optical beam reducing system just in front of the FSI, since the maximum input diameter beam that could be handled by the FSI was about $10 \mathrm{~mm}$. Two alternative ruggedized and temperature-stabilized Invar Questar telescopes, each combined with an 85-mm focal 1ength collimating lens, were used for this purpose. Diameters of 178 and $89 \mathrm{~mm}$ were employed for these two telescopes and they were each coupled to an 85-m $\mathrm{fl}$ collimator lens to produce (the same for each) an output beam diameter of $6.8 \mathrm{~mm}$. A photograph of the FSI together with the 178-mm Questar telescope is shown in Fig. 10 located on the optical bench in aircraft \#2. The exit window is on the right and the light path from the Questar to the window area was shielded by a tube (to exclude internal air turbulence), as shown in this photograph.

The laser source located on one aircraft was diverged (roughly to 15 to 30 mrad) and propagated through the window of this airplane to irradiate the window of the second airplane, and be subsequently collected by the Questar telescope. On transmission through the source aircraft window, the beam diameter was very small and was not influenced by the boundary layer at this window. On reaching the receiving airplane, however, the beam was large (roughly 15 to $30 \mathrm{~m}$ in diameter) and the receiving optics collect a bean equal in diameter to either the 89- or 178-mm aperture, according to whichever Questar optics was used.

The boundary-layer turbulence at this receiving optics window degraded the beam. Therefore the boundary-1ayer MTF measurement made refers to that aircraft on which the measuring (FSI) system was located, and the effective beam diameter was determined by the receiving optics diameter. 


\section{KC-135 AIRFOIL EXPERIMENTS}

This third and last set of measurements was developed as a culmination of the earlier flight experiments. A more comprehensive series of flight optical boundary-layer degradation measurements very similar in prineiple to those carried out earlier on the Lear Jet was planned. The optical configuration was similar in concept to that carried out on the Lear Jet, except that an optical beam of 89-mm diameter was employed.

A schematic of the optical experiment is shown in Fig. 11. A photo of the FSI instrument, the 100-mm optical beam expander (stopped down to $89 \mathrm{~mm}$ for the measurements), and the HeNe laser source, together with the auxiliary optics (beam splitter, mirrors, and laser beam expander) is shown in Fig. 12. This equipment was mounted on an optical bench along the aircraft fuselage (near the window which was located at point $A, 22.9 \mathrm{~m}$ from the nose of the airplane). A photo of the airplane showing the location of the airfoil is shown in Fig. 13.

Thirty seconds of MTF data were recorded on FM magnetic tape. Data were taken at each flight condition for which shear directions were either along $\left(\psi=0^{\circ}\right)$ or perpendicular to $\left(\psi=90^{\circ}\right)$ the airflow direction (using the " $\mathrm{K}$ ' mirror wave-front rotator). The flight profiles were planned so that data could be taken either along a constant Reynolds number line, or alternatively along a constant Mach number line, as aircraft altitude was changed.

In making measurements during these flights, real-time focusing was done as each flight condition was changed. A Nicolet Model 1070 signal-averaging system installed in the airplane was used to look at the real-time-averaged MTF signals. The Tropel 100-mm beam expander was focused for the best (highest) MTF while looking at the average of up to 1000 MTF curves. In the earlier experiments this had not been possible, and focusing by viewing an ever-changing MTF curve observed on an oscilloscope had been rather difficult and tedious. A wave-front rotator on the front of the FSI permitted the MTF measurement to be made along any direction between $\psi=0^{\circ}$ (along the air flow in the boundary layer) and $\psi=90^{\circ}$ (perpendicular to the airflow in the boundary layer).

Focusing was initially optimized for the $\psi=0^{\circ}$ direction and checked for the $\psi=90^{\circ}$ measurement. It was noticed that after focusing on the $\psi=0^{\circ}$ position, then going to the $\psi=90^{\circ}$ position (also some intermediate settings for $\psi=45^{\circ}$ were done), a refocusing of the optics was sometimes necessary to optimize 
the MTF. Apparently a small amount of residual astigmatism arose and one reason for this could be attributed to a small distortion in any one of the mirrors - the airfoil mirror or the turning mirror in the airplane on the optical bench. It was therefore necessary to ensure that temperature effects or flight-induced stresses from the mounting of these mirrors did not introduce distortions during flights.

In all these experiments, the test window consisted of a double (thermopane) window (Fig. 11), (while only a single window had been used in the earlier experiments), to minimize the temperature gradient effects in the window. The outer window was flush with the skin of the airplane. The airfoil mirror was flush with the airfoil surface within $1 \mathrm{~mm}$ and was located $60 \mathrm{~cm}$ from the skin of the airplane so that it essentially was outside the effective boundary layer. The optical path inside the airplane had also been carefully shielded from internal thermal air currents by a plastic sheet on all flights. An additional test carried out was to make measurements for a range of different aperture sizes, from 25 to $89 \mathrm{~mm}$ for two constant altitudes and one constant Mach number.

A great deal of effort was spent on testing all the optical components, mirrors, and windows, since these were more critical than in any of the earlier experiments due to the larger aperture beam making a double pass through all the components. Calibration measurements were made on the ground (to the airfoil) and in flight (with an internal aircraft mirror) as well as in the laboratory. This was needed to both check out the optics and to accurately determine the shear scale. In the experiments with the 89-mm aperture, the beam size entering the interferometer was over $9 \mathrm{~mm}$ (which is somewhat larger for visible wavelength than was the case in all the $\}$ earlier experiments). At the higher shear values for the visible FSI, the shear scale is nonlinear by up to 7 or 8 percent. Therefore, this nonlinearity was corrected during the data reduction and computer processing. Note that this correction depends on the properties of the shear plate, its refractive index and thickness, and in none of the earlier experiments was it necessary to make this correction. 


\section{FLIGHT DATA RESULTS}

A11 the data were computer processed to yield averaged MTF data, and standard deviations of MTF, the processed averaged MTF data obtained for all these experiments were next fitted to the theoretical "Barakat" expression and the equivalent optical rms wavefront deviation $(\sigma)$ in wavelengths (for $\lambda=632.8 \mathrm{~nm})$ and correlation length $(l \mathrm{~mm})$ factors calculated for the series of data reported here. The values of $\sigma$ and $\ell$ for the different experiments were then plotted as a function of altitude.

In the case of the Lear Jet and the airfoil experiments, the $\sigma$ evaluated from the MTF data corresponds to double passes through the boundary layer. In order to compute the equivalent single pass, it is necessary to know how $\sigma$ varies with path length. If the refractive index fluctuations for a double pass through the boundary layer can be assumed on the second pass (assuming the time of propagation is very much smaller than the fluctuation time of the refractive-index variations) to be correlated, then it will be assumed that the single-pass $\sigma$ value is just half the double-pass $\sigma$ value. This point has been recently discussed by Hogge and Gilbert. ${ }^{23}$ D'Amato described the development of an aerodynamic model ${ }^{12}$ and gave a theoretical analysis of the characteristics of the boundary layer to show how calculations of the density fluctuations could be made from the boundary-layer parameters. It was necessary to make a number of assumptions in applying his aerodynamic model, taking as a starting point a standard atmosphere and extrapolating from the known properties of the flow over a simple flat plate.

The experimental results encompass a wide range of test conditions in which the aerodynamic parameters of the boundary layers can be expected to vary by substantial amounts. A comparison of the experimental data with the predictions from D'Amato is shown in Figs. 14 and 15 (a) and (b). The points (shown by open symbols as indicated on the figure) computed by D'Amato ${ }^{12}$ are plotted for many of the corresponding experimental points. For the KC-135 airfoil test data, points derived from some NASA aerodynamics measurements (see K. Gilbert, Reference 24, on page 163) are shown compared to the optical measurcments in Fig. 14(c). For the propagation experiments, the measured data showed little or no variation with airplane speed, and only the averaged data point over all Mach numbers is shown. The amplitude of typical error 
bars which denote the standard deviation of the mean value taking the average of 40 separate MTF measurements is shown on the figures, using that data computed from the standard deviation of a single reading for the MTF.

The Lear Jet and propagation experimental data are significantly more degraded than D'Amato's values. The airfoil data $\sigma$ value points show a reasonably good agreement with the points derived from the aerodynamic measurements. The Lear Jet and propagation experiments were flown in late January and between the winter months of September and March, during which ambient temperatures were appreciably colder at most altitudes. The airfoil experiments were carried out in June and July, and were the only case where detailed aerodynamic data were taken (by NASA Ames). Actual measurement data were not available for these factors to be taken explicitly into account in the D'Anato computations. However, some estimates of the sensitivity to temperature changes (which will produce corresponding changes in the boundarylayer turbulence) have been discussed by D'Amato and indicate that ambient temperature may play a critical part in these computations. It should be pointed out that in the earlier cube corner interferometer experiments a marked increase in degradation was observed as the airplane flew at constant altitude and speed into a cold front. ${ }^{7}$ At this point, while the D'Amato theory appears to be able to make predictions of the degradation, only by making the aerodynamic measurements can it be expected that good agreement of calculated with measured optical data will be obtained.

There are several details in the comparison shown in Figs. 14 and 15 that should be enlarged upon for clarity. The Lear Jet data shown in Fig. 14 (a) relate to a $25-\mathrm{mm}$ optical diameter and for this reason; as well as the boundary layer itself being much smaller, these data are less degraded than the propagation and airfoil data in Figs. 14 (b) and (c). These later experiments were taken mostly with an 89-mm-diameter beam (except where indicated differently) on a KC-135 airplane with a thicker boundary layer than on the Lear Jet. In the final analysis, it is the absolute values of $\sigma_{0}$ and $\ell_{0}$ of the boundary layer that must be determined, and these are readily scaled for a given diameter optical beam to the corresponding $\sigma$ and $\ell$ values in order to compute the optical degradation with any given optical aperture. 
The Lear Jet data showed only small variation with Mach number, except at $1.5 \mathrm{~km}$ altitude where a large difference for 0.4 and 0.55 Mach number was observed. The theoretical calculations at all altitudes showed little change with Mach number so that at $1.5 \mathrm{~km}$ altitude, there is an additional difference between theory and experiment in the magnitude of the dependence on aircraft speed.

For the propagation data, two theoretical points are shown at each altitude (for two different Mach numbers) representing the slowest and fastest speed flown at that altitude. In these cases the theoretical computations refer to the case of the boundary layer at the aft location on the KC-135 aircraft. In Fig. 14(b), the theoretical points indicated at each altitude are different for the two limiting aircraft speeds (minimum and maximum for the experimental data taken). In the experimental data, these differences are hidden in and less than the spread of the data points experimentally observed. However, in taking data at different Mach numbers at a constant altitude, the environmental changes in the aerodynamical conditions were not as large as when changing altitude, and the spread of data was generally less. Both variations in the boundary-1ayer characteristics due to the changing flight environment as well as experimental variation in the data may therefore account for these differences. These disparities are not a serious discrepancy, but provide some indication of the complexity of the interaction of the aerodynamic parameters in the turbulent boundary-layer problem.

Comparison of the correlation lengths shown in Fig. 15 from theory and experiment, shows good agreement for the Lear Jet, but less so for the propagation data. The two low-altitude measured data with long correlation lengths (greater than $30 \mathrm{~mm}$ ) are exceptions and may indicate that the measured degradation here was not solely due to the boundary layer. For the KC-135 airfoil experiment, D'Amato's calculations predicted correlation lengths of the order of $20 \mathrm{~mm}$, and similar correlation lengths were also obtained from the aerodynamic data (selected data from Ref. 24 are shown in Fig. 15(c). All the other data for the $\mathrm{KC}-135$ airplanes yield experimentally measured correlation lengths slightly shorter than predicted by theory. The disparities are most likely mainly due to the uncertainty in the aerodynamic environmental conditions and parameters. 
In Figs. 16 and 17 the aperture scaling measurements from the airfoil data are compared with the theoretical predictions for optical beam diameters of 89,50, and $25 \mathrm{~mm}$, taken at constant Mach number (0.57) and for two constant altitudes. All the data at each altitude were gathered within an approximately 30-min time interval, and environmental conditions were expected to be relatively constant. The lower altitude was flown first, and in this case good agreement between the measured and predicted data points can be observed. At the higher altitude, the measured data were more degraded than expected (when compared with data for the 89-nm aperture taken on earlier flights), and for all aperture sizes the $\sigma$ values measured were larger than predicted by theory.

The spread in the measured data over the $25-$ to $89-\mathrm{mm}$ aperture diameters in question, is much smaller at both altitudes than the predictions from theory, and the uncertainties in the aerodynamic quantities are suspected of being the source of the error in this case. There is no doubt that the variations in environmental conditions produce large spreads in the data. 6. CONCLUSIONS

When this work was started, there were no detailed experimental or theoretical results that could provide realistic estimates of the amount of degradation to be expected from an in-flight aircraft boundary layer. The initial emphasis was placed on obtaining experimental measurements of the flight boundary-1ayer degradation together with doing a preliminary optical analysis. This has subsequently been followed by a more detailed aerodynamical analysis (by D'Amato) making use of these measured data. ${ }^{12}$

A description of these experiments together with an outline of the optical analysis has been discussed here, and the measured data compared with some of the theoretical predictions in the case of the boundary-layer degradation. There are many factors that enter into each one of these series of tests. Intermittent fluctuations in the environmental conditions caused changes in the experimentally measured data for a given flight condition. Each series of tests used different geometric configurations, and effects due to the airfoil structures, and due to airplane geometry itself, may give rise to environmental changes and effects. The theoretical calcu1ations of D'Amato were based upon estimated "steady state" conditions since no experimentally measured aerodynamic data were available. 
The boundary-layer degradation measured in these experiments varied with altitude, airplane speed, and Reynolds number, in a complex way, and also depended on the ambient temperature. Data obtained from experiments conducted over the winter months were more degraded than expected. The Lear Jet data, where the boundary layer was significantly thinner than in the later tests (and also were taken using smaller diameter optics), were less degraded than the later tests. (The boundary-layer thickness in the $\mathrm{KC}-135$ was from 3 to 5 times thicker than the Lear Jet.) There were also different boundary layers for the two window locations on the two different aircraft (aft and forward positions). However, there were only small differences between the results for these two cases, the forward position (thinner boundary layer) giving rise to more degradation than the aft position.

To summarize, the measured results indicated degradation levels for the $\mathrm{KC}-135$ airplanes between $\sigma=0.10 \lambda$ to $0.13 \lambda$ increasing to $0.18 \lambda$. When scaled to infinite aperture, these correspond to degradation levels of $\sigma_{0}=0.2 \lambda$ to $0.26 \lambda$ and increase to $0.36 \lambda$. For the Lear Jet, degradation with a $25-\mathrm{mm}$ diameter optics was roughly $\sigma=0.07 \lambda$ and on scaling to infinite aperture, corresponds to a degradation leve1 of $\sigma_{0}=0.14 \lambda$ which is less than that observed on the $\mathrm{KC}-135$ as was expected. The corresponding measured correlation lengths of roughly $12 \mathrm{~mm}$ for the $\mathrm{KC}-135$ aircraft and $6 \mathrm{~mm}$ for the Lear Jet scale to roughly 20 and $25 \mathrm{~mm}$, respectively, for infinite apertures. These boundary-1ayer correlation lengths do not appear to reflect the different boundary-layer thicknesses on the two different aircraft. To interpret the data and to extrapolate and predict the final imaging characteristics, the appropriate aperture scaling must be taken into account. There is clearly no simple rule-of-thumb answer to estimating the degradation in a light beam propagated through the aerodynamic boundary layer of an aircraft. However, in those cases when aerodynamic measurements are made, these show good agreement with optical measurements. 


\section{REFERENCES}

1. H.A. Stine and W. Winovich, 'Light Diffusion Through High Speed Turbulent Boundary Layers," NACA-RMA 56 B21 (May 1956).

2a. R.E. Hufnage1, "A Theory for the Image Degrading Effects of Turbulent Aerodynamic Boundary Layers," Engineering Report 7677, Perkin-Elmer Corp. (6 May 1964).

2b. R.E. Hufnage1 and R.V. Shack, "The Effective Transfer Function of a Turbulent Boundary Layer," Engineering Report 7673, Perkin-Elmer Corp. (1964).

3a. G.W. Sutton, "On Optical Imaging Through Aircraft Turbulent Boundary Layers," Avco-Everett Report AMP334 (June 1971).

3b. G.W. Sutton, AIAA J. $\underline{7}, 1737$ (1969) .

4. A.M. Veed and J.W. Tuttle, "Derivation of an Optical Modulation Transfer Function for Turbulent Boundary Layers," General Dynamic Report ERR-RW-347 (29 September 1964).

5. C.A. Luke, "Atmospheric Turbulence MTF Measurements for Airborne Surveillance Techniques," Technical Report AFAL TR-68-253, Part II, Air Force Avionics Laboratory (May 1969).

6. B.W. Bryant, "Experiments on Airborne Seeing from a High Altitude Jet Aircraft," Project Report AOR-29, Lincoln laboratory, M.T.T. (18 September 1973), DDC AD-896818-L.

7. D. Kelsa11, JOSA 63, 1472 (1973).

8. D.J. Wolters, "Aerodynamic Effects on Airborne Optical Systems," Report MDCA2582, McDonne11 Aircraft Co., St. Louis (14 December 1973).

9. W.L. Visinsky, "Boundary Layer Turbulence," Air Force Weapons Laboratory, Laser Division Digest LRD-72-1, (June 1972). p. 109.

10. AFWL/Optics Semiannual Technical Summary, Lincoln Laboratory, M.I.T. (15 March 1973), P. 13, DDC AD-526201-L.

11. K. Gilbert, D.F. Terwilliger, R.J. Cook, "Laser Propagation Through an Aircraft Turbulent Boundary Layer: Lear Jet Experiments," Air Force Weapons Laboratory, Laser Digest TR-75-229 (October 1975), p.143.

12. R. D'Amato, AFWL/Optics Final Report, "Optical Degradation by Aerodynamic Boundary Layers-Part II," Lincoln Laboratory, M.I.T. (30 September 1977). 
13. D. Kelsall, Appl. Opt. 12, 1398 (1973).

14. D. Kelsal1, (a) Proc. SPIE 46, 124 (1974), (b) Phot. Sci. Eng. 21, 123 (1977).

15. H.H. Hopkins, Opt. Acta, 2, 23 (1955).

16. H.H. Hopkins, Proc. R. Soc. 231, 91 (1955).

17. M. Born and E. Wolf, Principles of Optics (Pergamon Press, New York, 1964).

18. E.L. O'Neill, Introduction to Statistical Optics (Addison-Wesley, Reading, Mass., 1963).

19. R. Barakat, Opt. Acta, 18, 683 (1971).

20. C.B. Hogge, AFWL internal communication, "Random Phase Variations Effect on the Strehl Ratio" (23 September 1977).

21. R.J. Cook, AFWL internal communication, "Comment on MTF Data Analysis" (1976).

22. L.D. Weaver, Lincoln Laboratory, M.I.T. internal communication, "Ca1culation of the Average OTF in the Presence of Random Fluctuations"

(31 March 1976).

23. K. Gilbert, "Thermo-optica1 Turbulent Boundary Layer Measurements," Air Force Weapons Laboratory, Laser Digest, AFWL TR-78-15 (April 1978), p. 140 .

24. K. Gilbert, "Aircraft Aero-Optical Turbulent Boundary-Layer/Shear-Layer Measurements," Air Force Weapons Laboratory, Laser Digest, AFWL TR-78-15 (April 1978), p.154. 


\section{ACK NOWLEDGMENTS}

The work degcribed in this report was carried out by a team of M.I.T. Lincoln Laboratory personnel at the Albuquerque Airborne Optica Site (AAOS) operated by Lincoln Laboratory at Kirtland Air Force Base. New Mexico, together with a wide range of support from many different particlpants who have contributed in one way or another to this program. In particular, I am grateful to E. J. Casaza and J. R. Theriault for their help during the earlier laboratory and field testing experiments. R. A. Bollman, R. G. Garnes, L. W. Chaulk, D. M. Corbostero, D. A. Page, J. Swedberg, and Dr. L. D. Weaver at AAOS played the major part in planning, designing, bulding, and teating of the experimental setupe, and in the overall fight measurements program. The development of the data handling, data reduction, and the Implementation of the computer analytical procedures was carried out with the assiatance initially of the Lexington Lincoln Laboratory Data Analyais Group, and later at AAOS by R. A. Bollman, G. Prade, and J. W. Tolleson (Lincoln Laboratory personnel) working with the Kirtland Air Force Computation Center during the fight experimental measurements phase of the program.

The help of General Dynamice (an Air Force contractor at AFWL) during the construction and aseembly of the Cycle II $\frac{1}{2}$ equipment, and for the use of aeveral opt1cal facilties, the assistance of the Air Force Optical Testing Facilities (run by International Lasers Syatems, Inc.) is also much appreciated. The continuous oupport and help provided by the Atr Force Weapons Laboratory Project Officera. Lt. Col. K. Gilbert, Captains G. Bock, J. Otten, and L. Pape, with whom the AAOs worked closely during the course of these experiments is gratefully acknowledged. In addition, mention must also be made of the help provided by NASA-Amea in the case of the Lear Jet experiments conducted from Moffet Field in Californla, and by the Air Force Might crews and support personnel who made the night expertments both posible and very successful.

Finally I am indebted to E.S. Cotton for both his support and for discusalons with him, aince the inception of this work, to $R$. D'Amato for his inputs and discuasions on the aerodynamics, and to Dr. R.H. Rediker for his comments. 


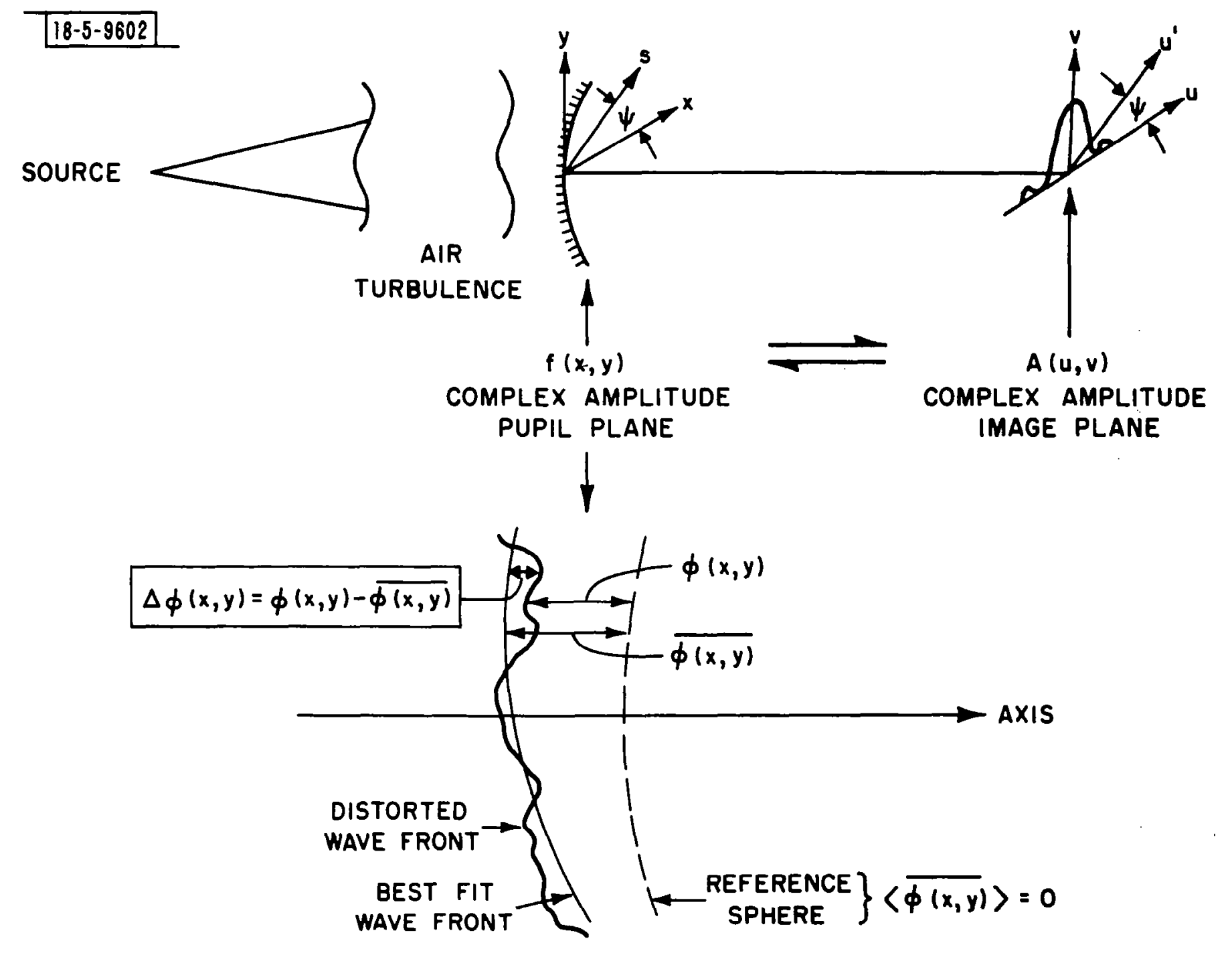

Fig. i. OPTICAL Relationships, 


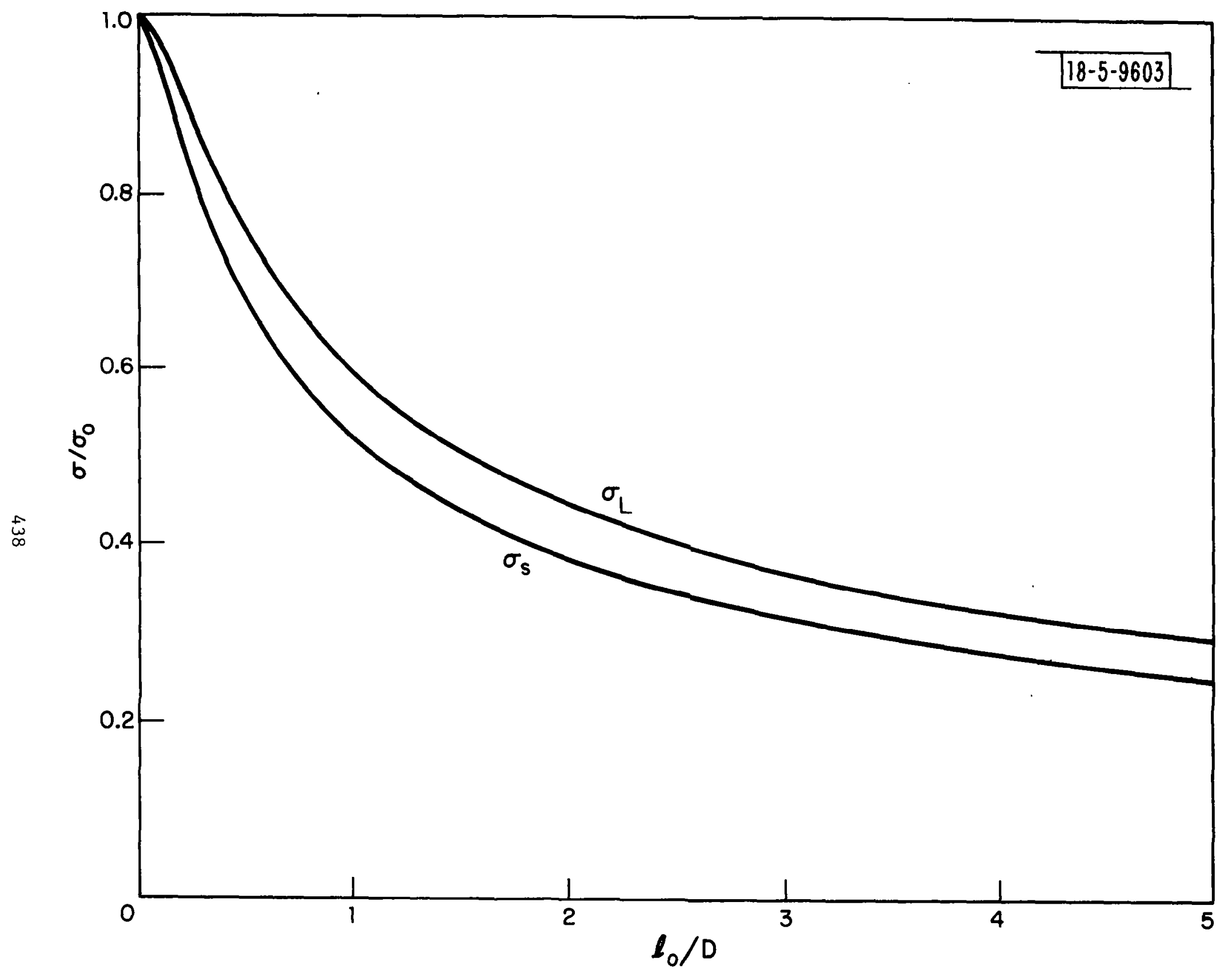

Fig. 2. Scaling of optical $\sigma$ with aperture (D) and correlation length $\left(l_{0}\right)$. 


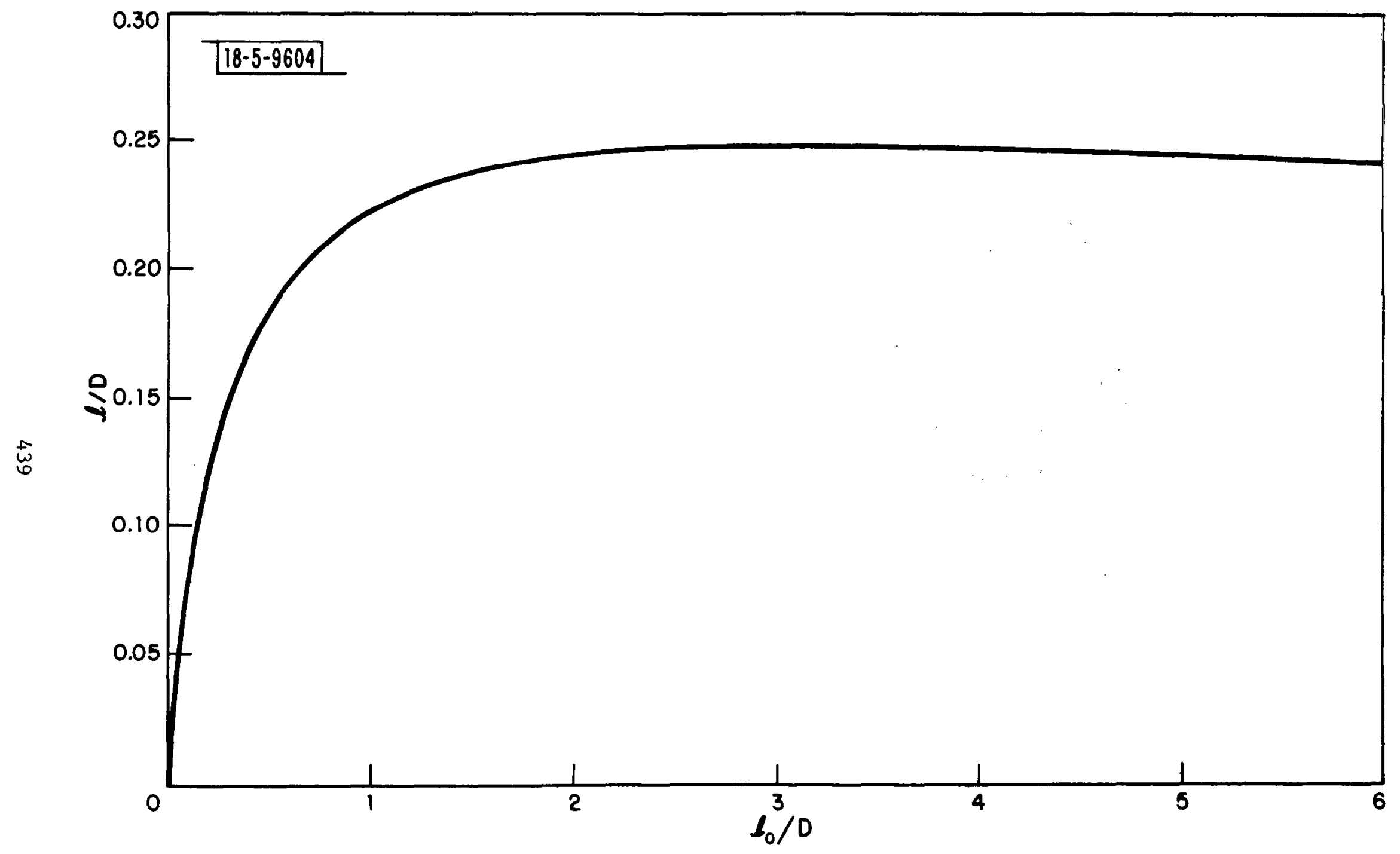

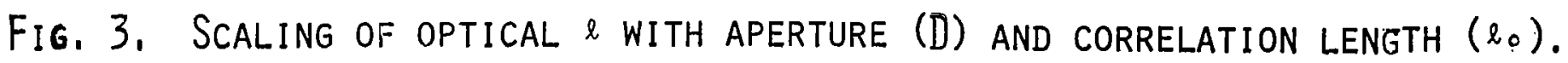




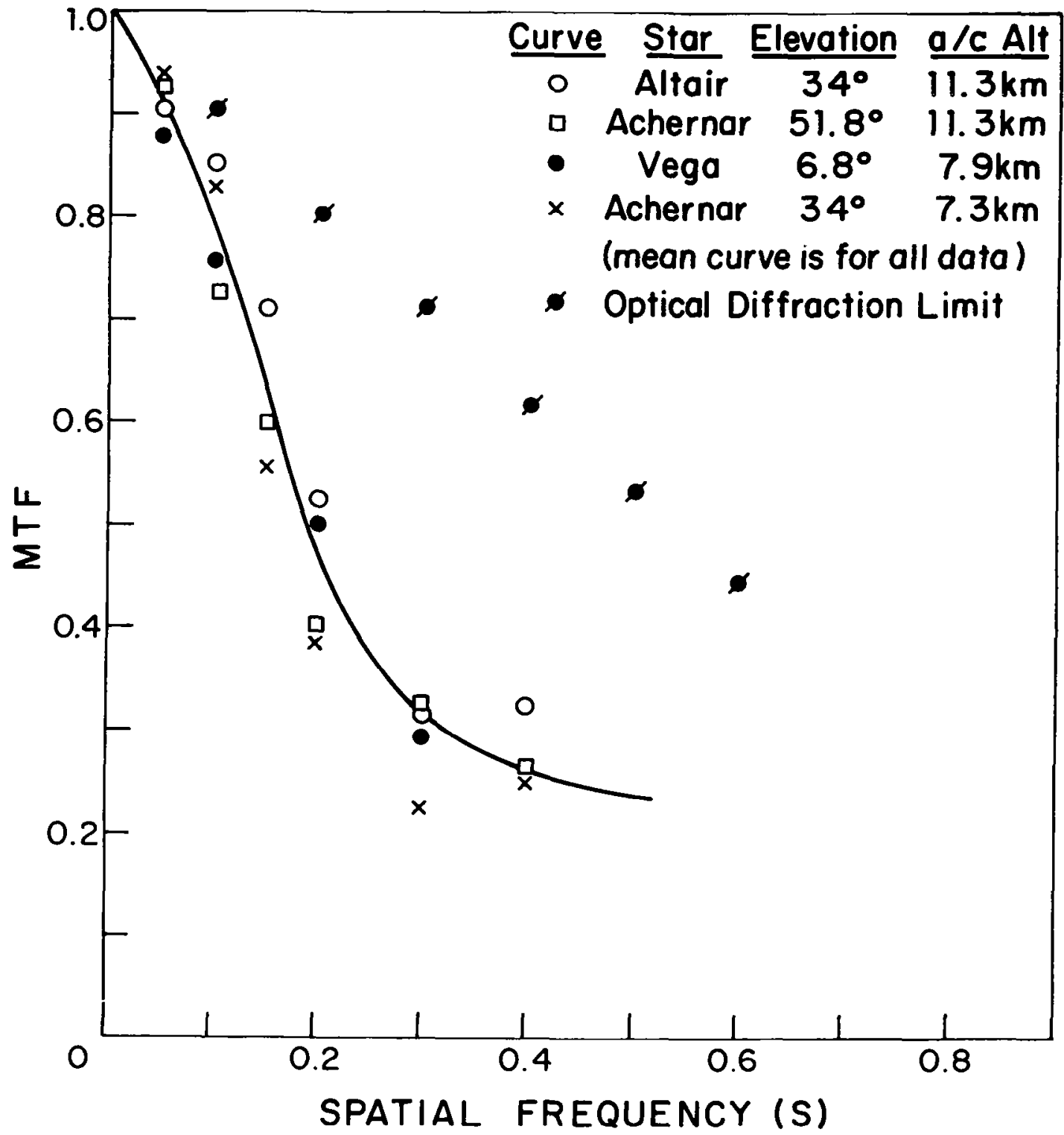

Fig. 4. MTF MEASUREMENTS FROM A KC-135 AIRCRAFT VIEWING STAR SOURCES. 


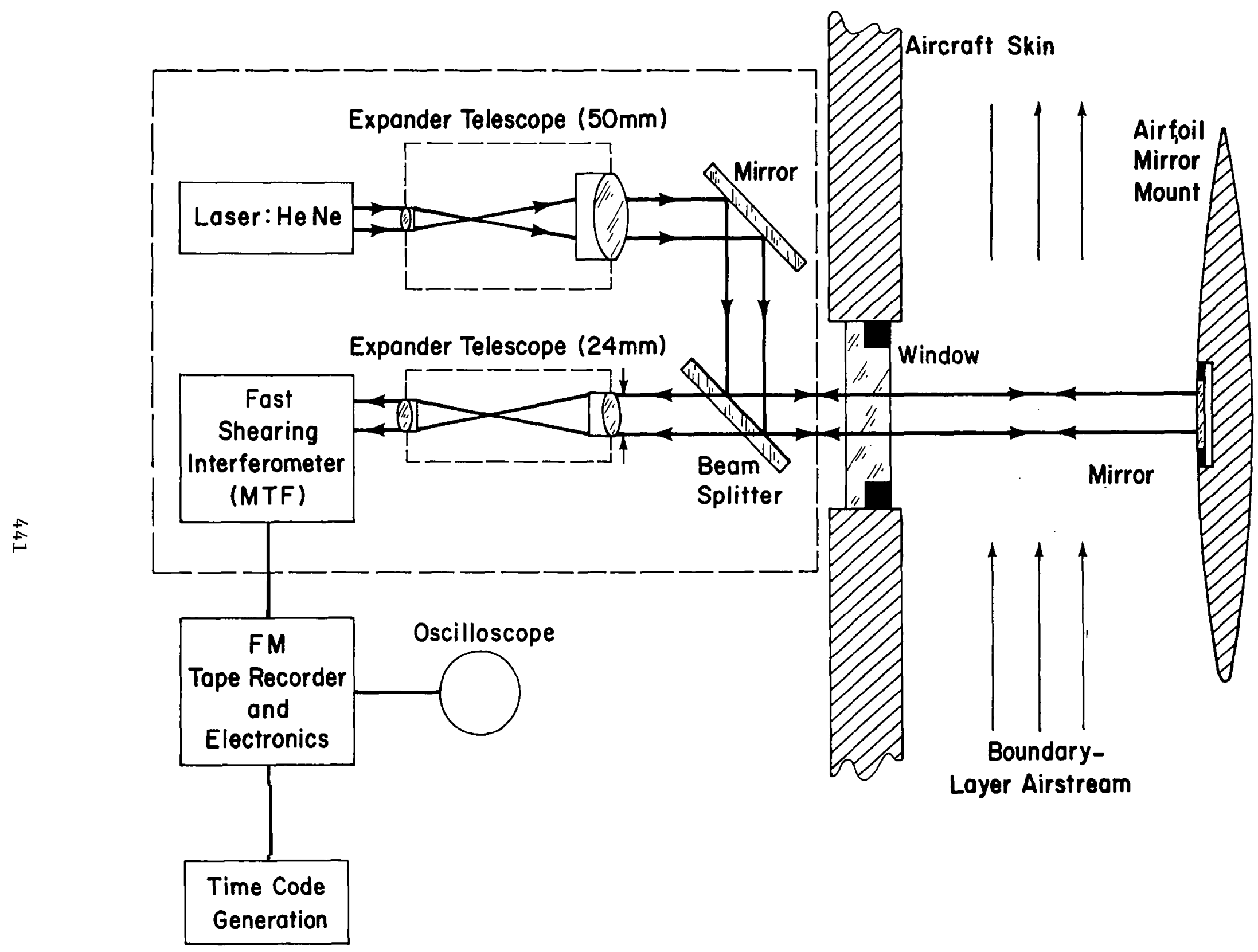

FIG, 5 OPTICAL SCHEMATIC LEAR JET EXPERIMENT. 


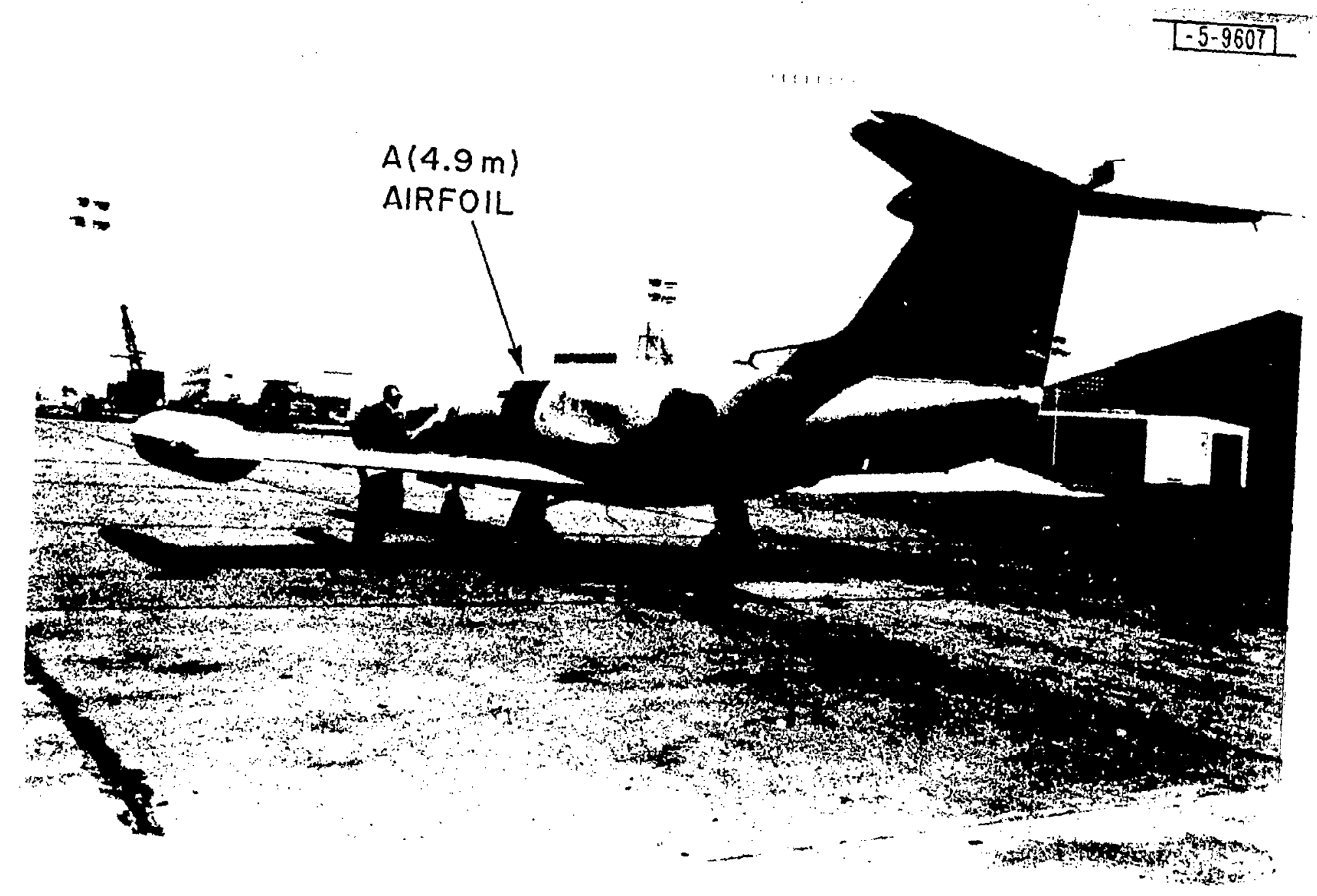

Fig. 6. LEAR Jet With aIRfoIl ON SIDE. 


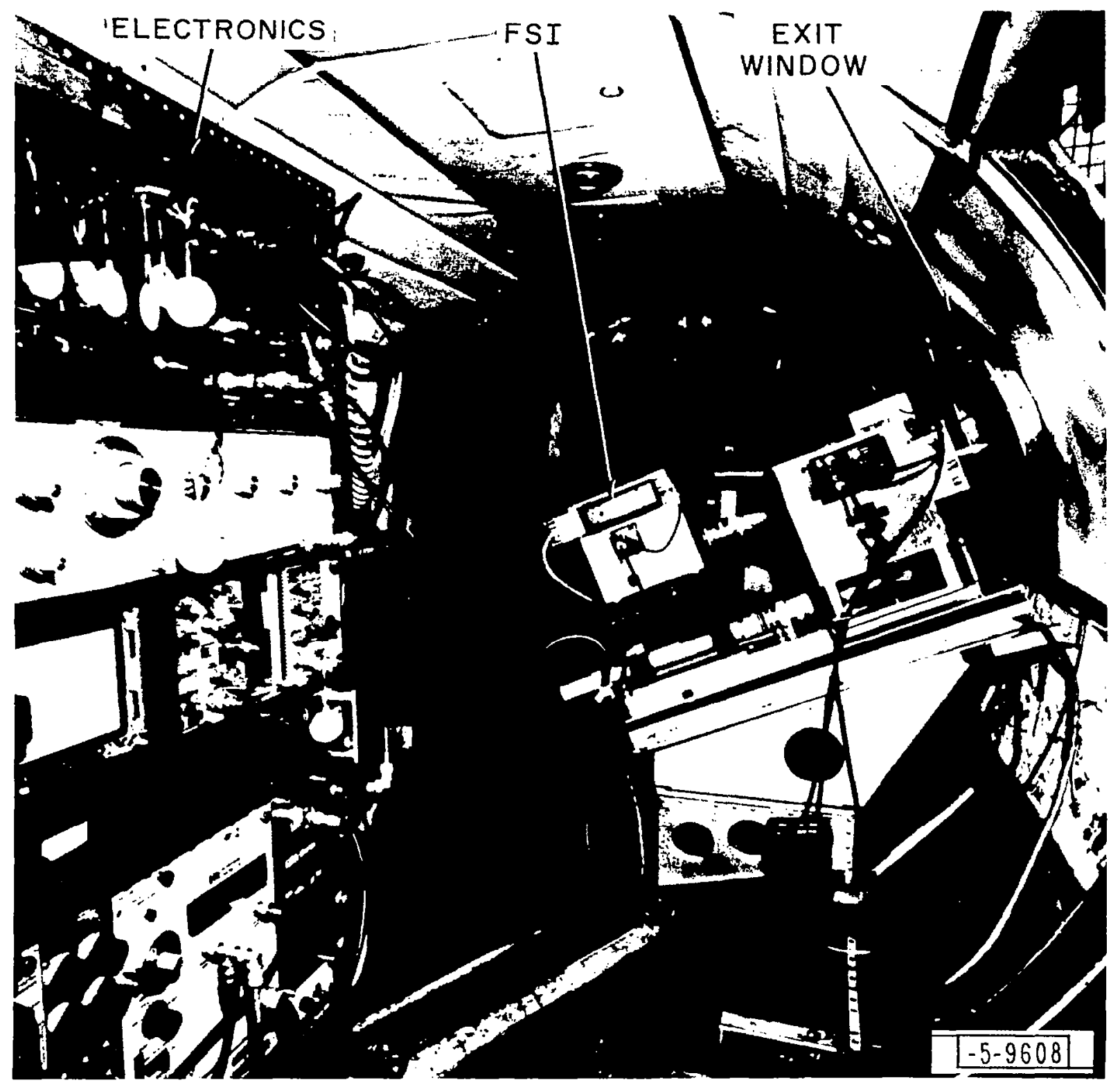

Fig, 7. FSI inside Lear Jet. 


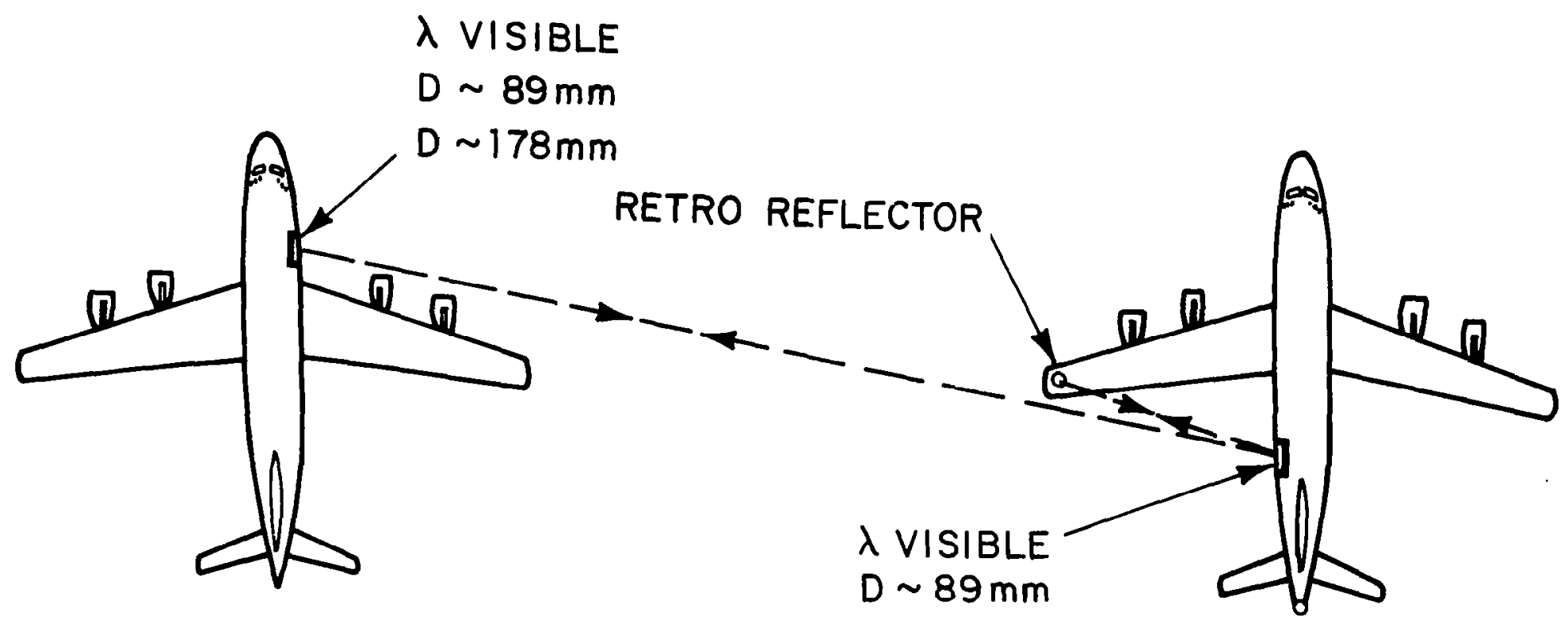

AIRCRAFT NO. 2

AIRCRAFT NO. 1

FIG. 8

AIRCRAFT PROPAGATION SCHEMATIC

FOR PROPAGATION EXPERIMENT

( $K C-135$ airplanes). 


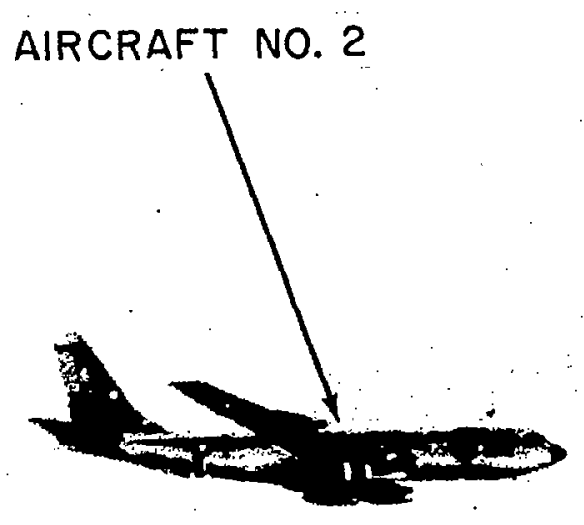

E

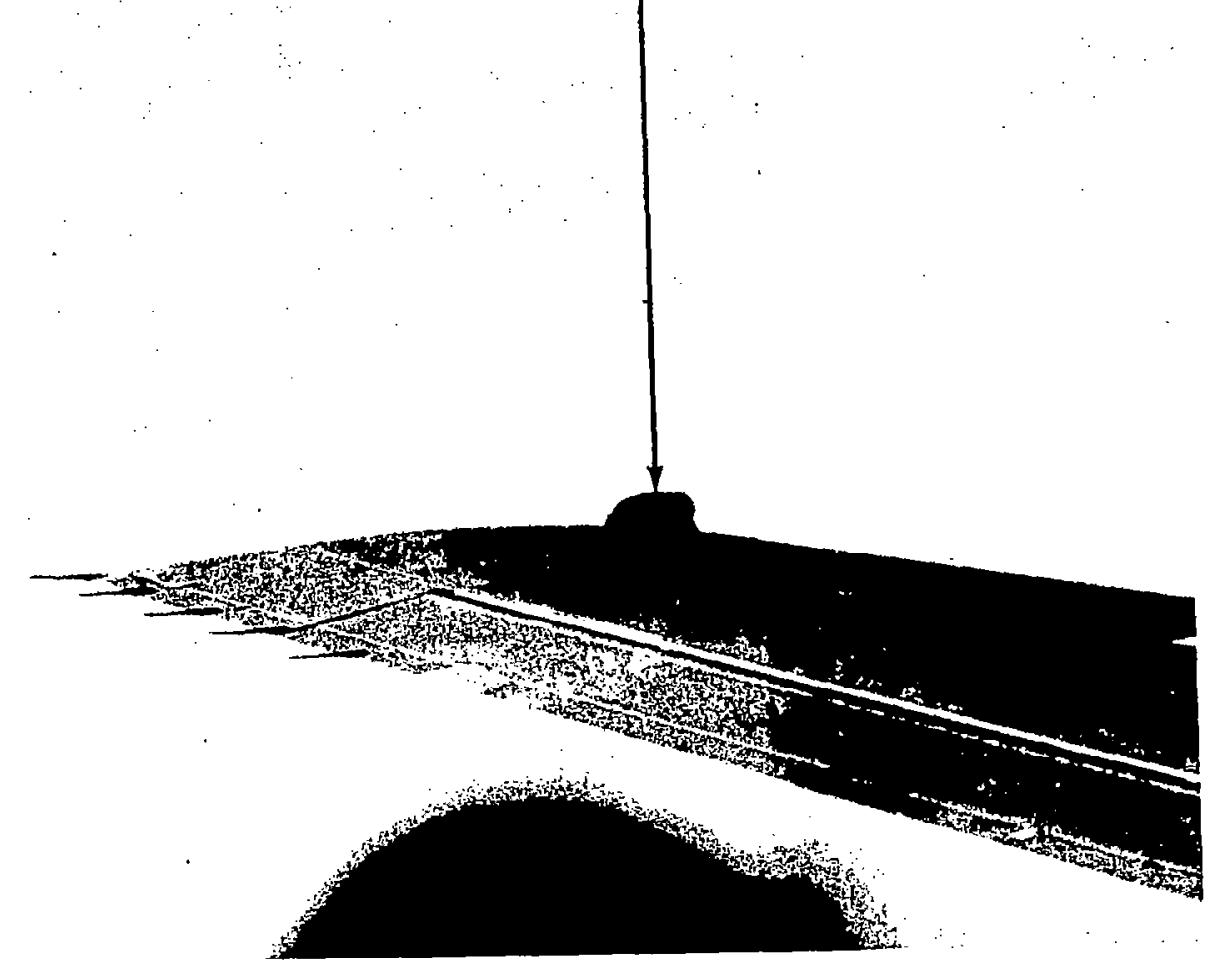

Fig, 9. Wingtip RETROREFLECTOR ON A/C :1 (WITH A/C :2 IN BACKGROUND), 


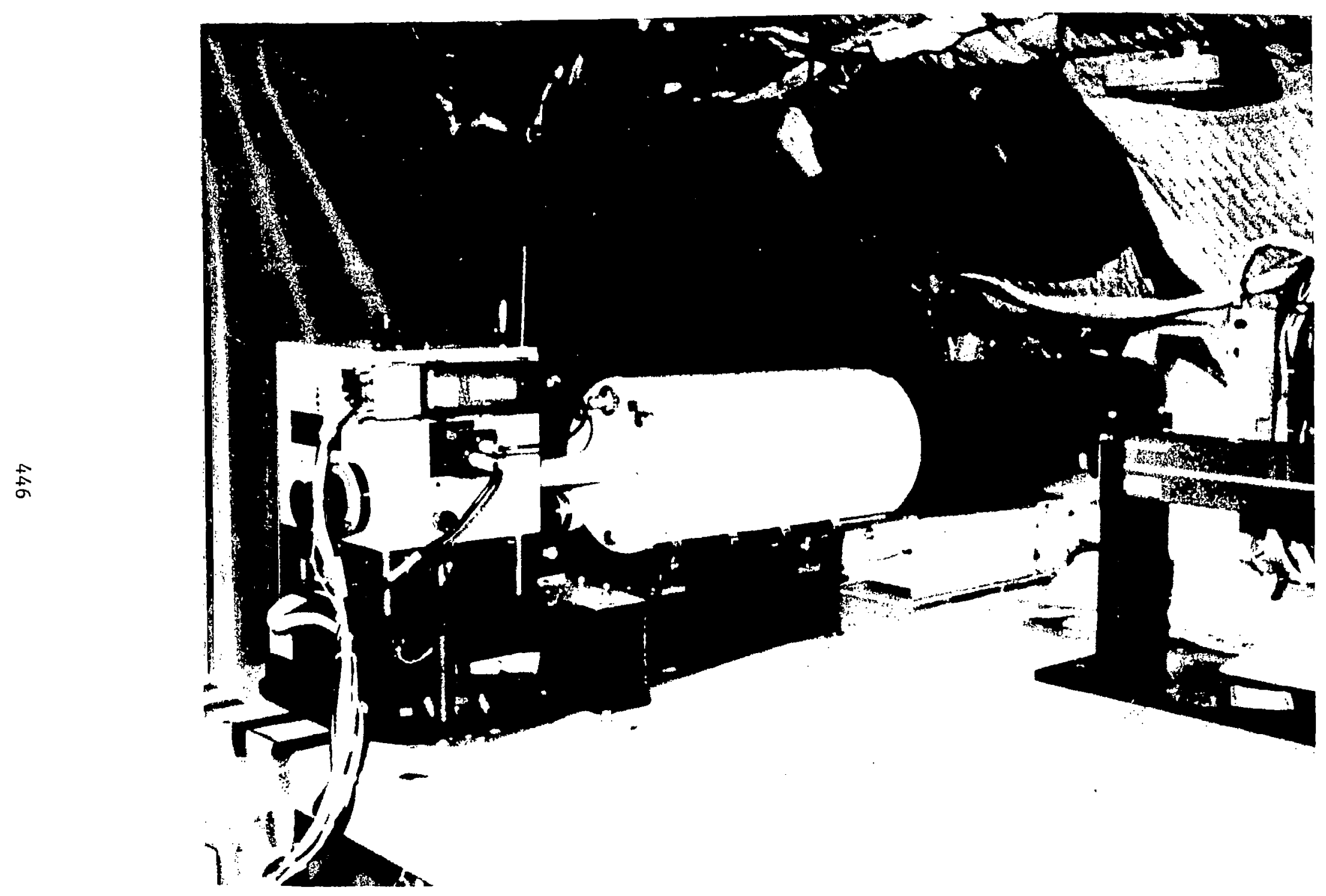

Fig, 1J FSI AND $178 \mathrm{~mm}$ OPTICS ON A/C NO.2 (propagation experiment). 
$18-00-13324-1$

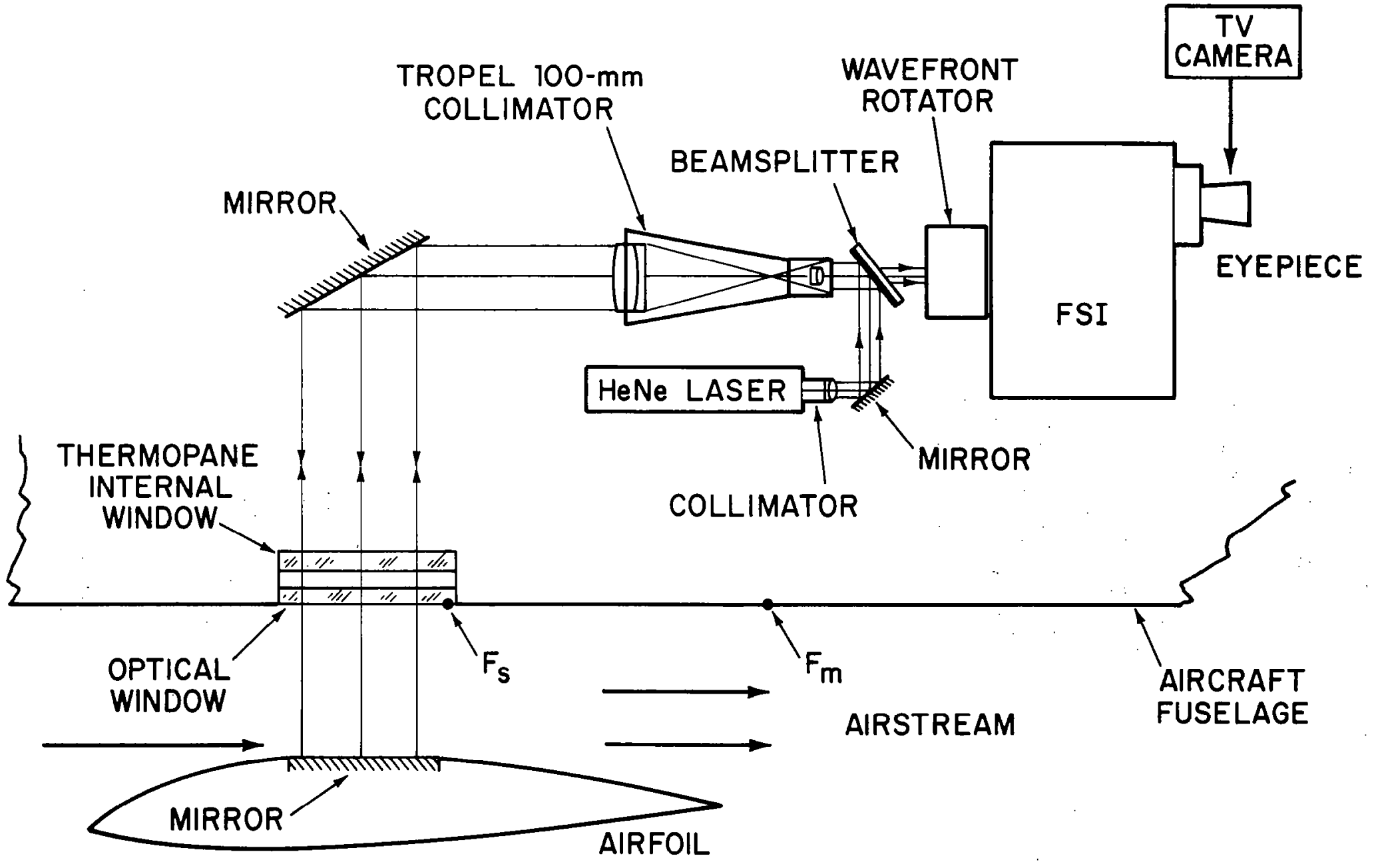

FIG, 11 OPTICAL SCHEMATIC KC-135 AIRFOIL EXPERIMENT. 


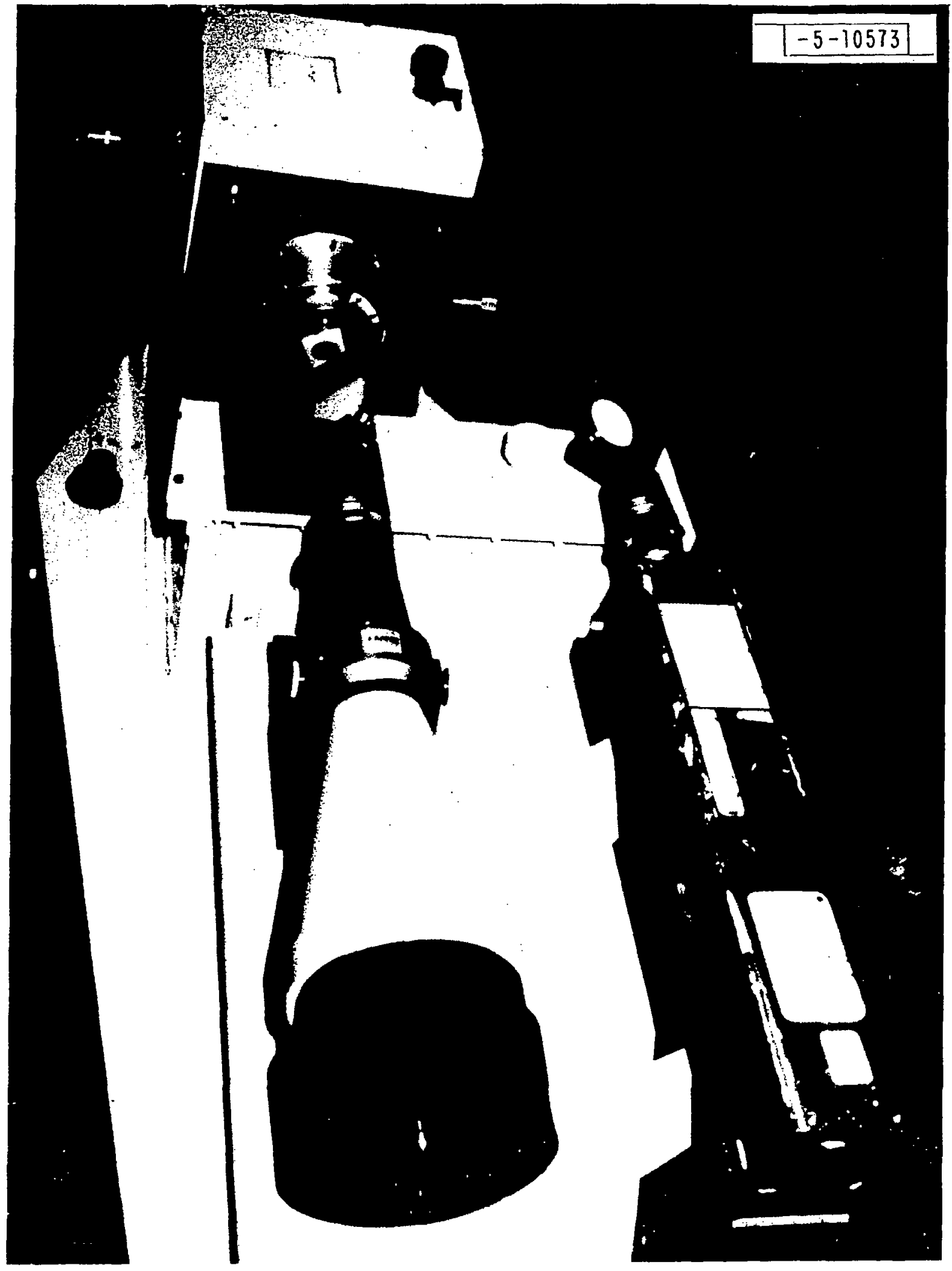

Fig. 12. FSI, 100-MM OPTICS, AND LASER SOURCE FOR KC-135 AIRFOIL EXPERIMENT. 


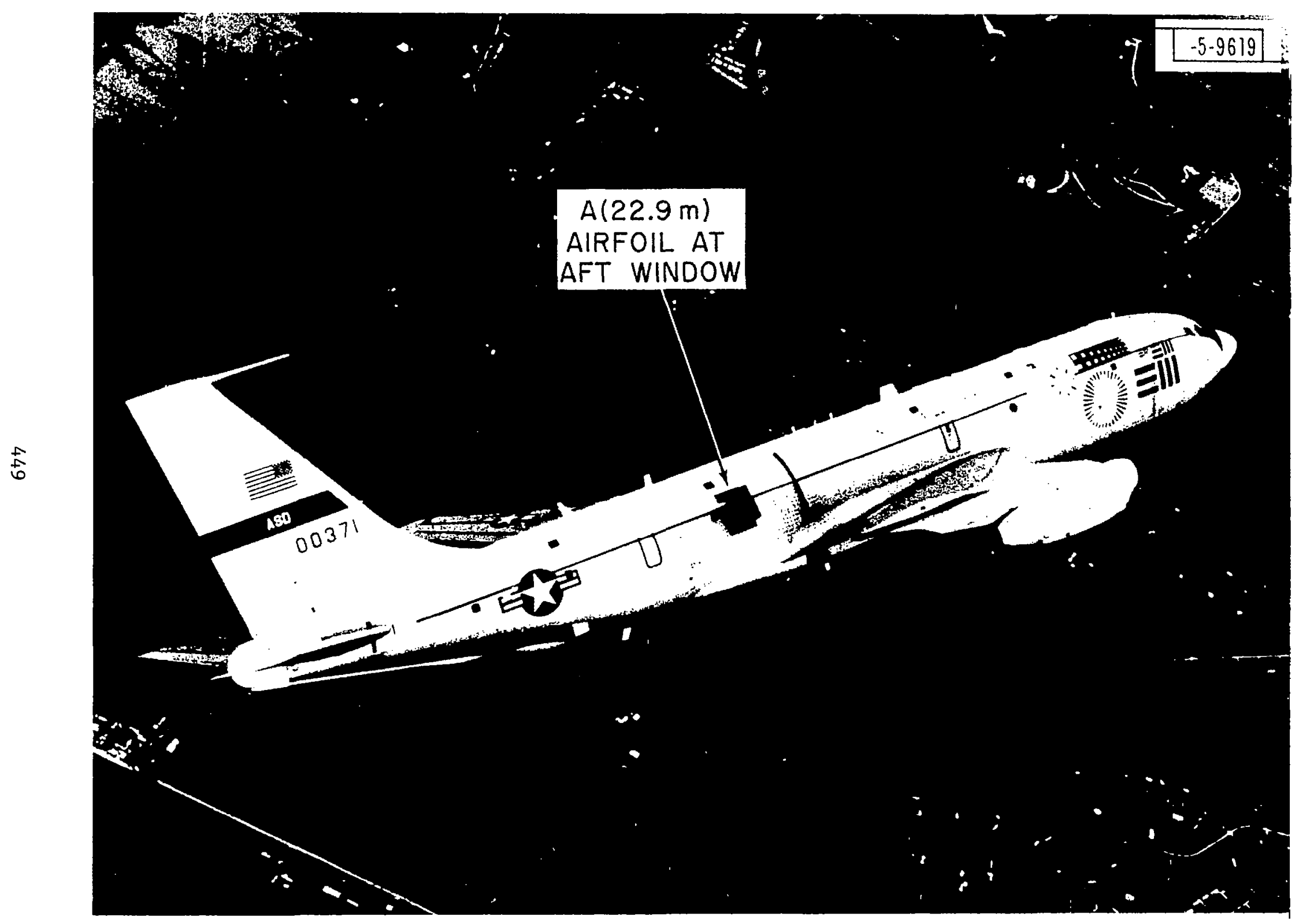

Fig, 13. AIRFOIL ON A/C 12 FOR KC-135 AIRFOIL EXPERIMENT. 


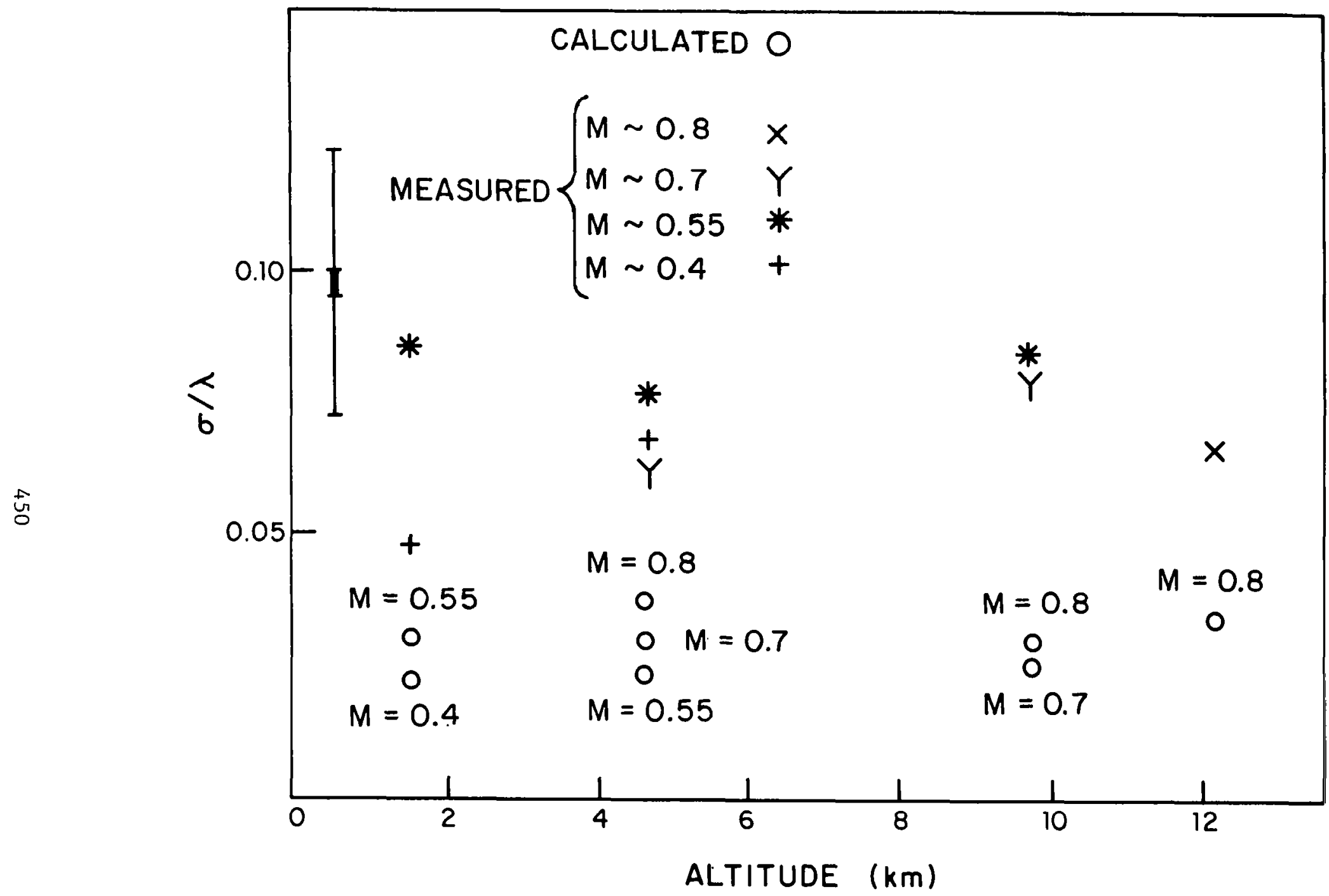

(a) LEAR JET.

FIG, 14 FLIGIT BOU:LA?Y LAYEI? 。 DATA. 
$18-5-9639-1$

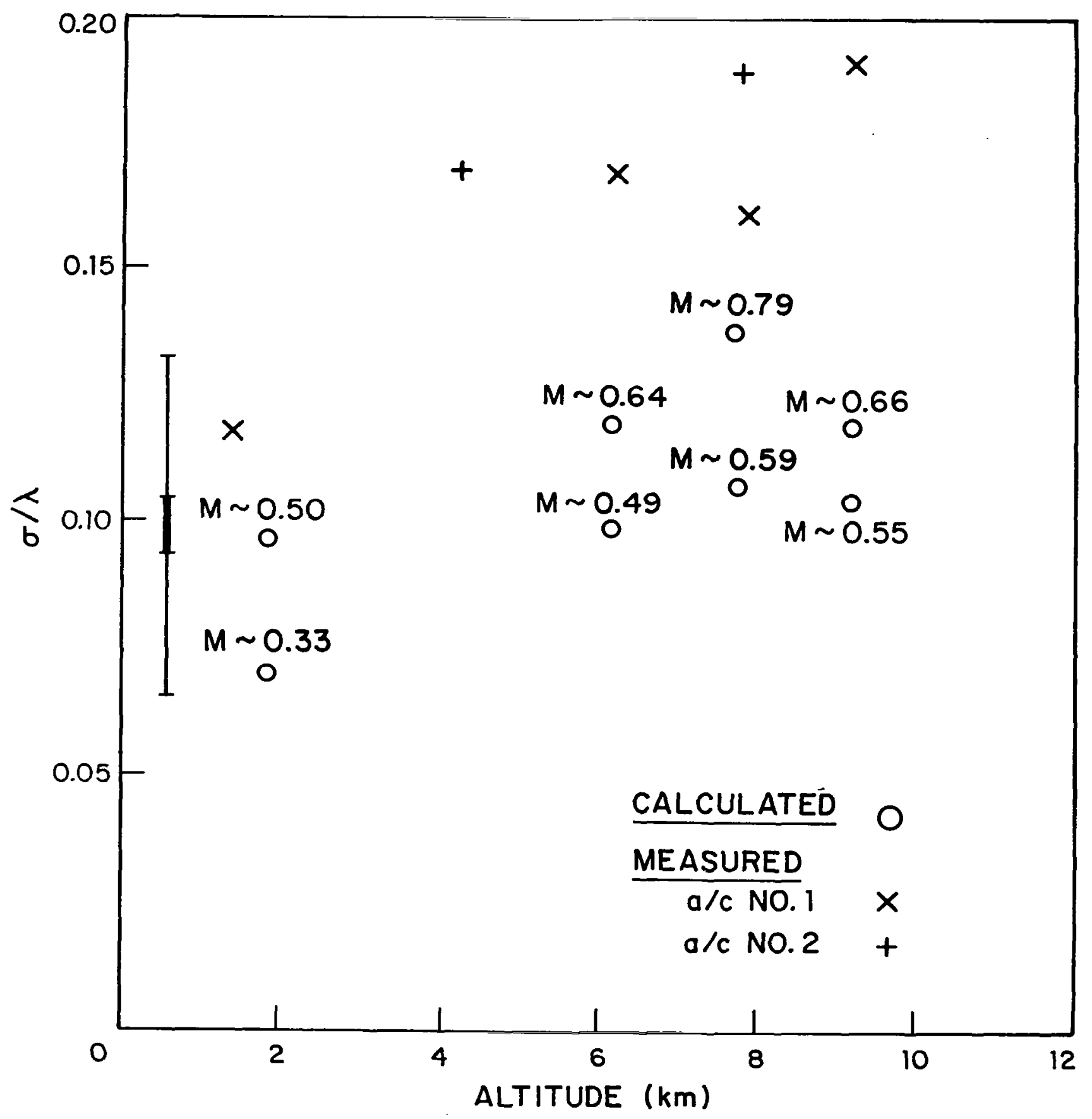

(b) KC-135 PROPAGATION EXPERIMENTS.

FIG. 14 FLIGHT BOUDANY LAYER $\sigma$ DATA. 


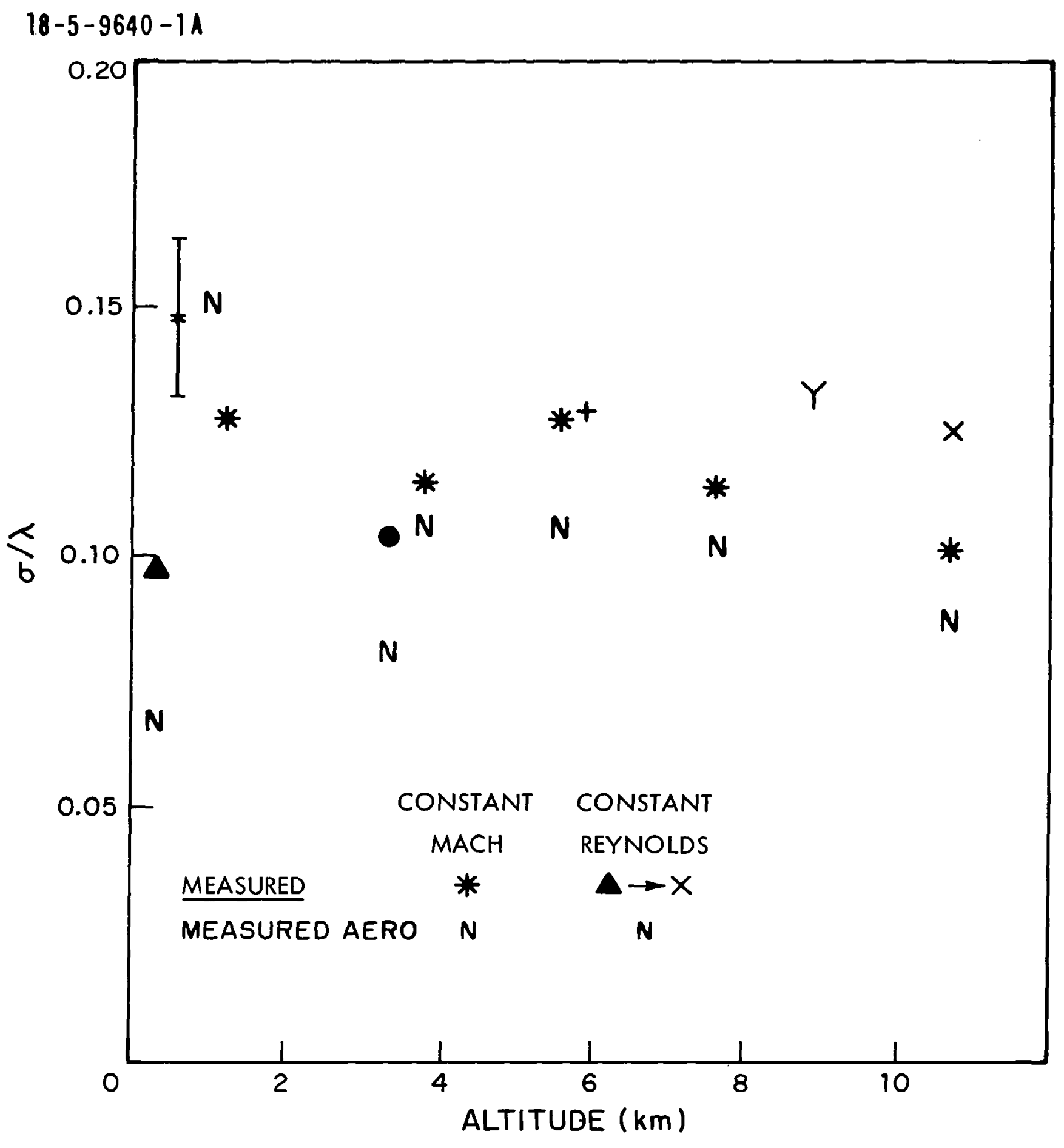

(c) KC-135 AIRFOIL EXPERIMENTS.

FIG, 14 FLIGIT BOUIDARY LAIE? O DITA. 


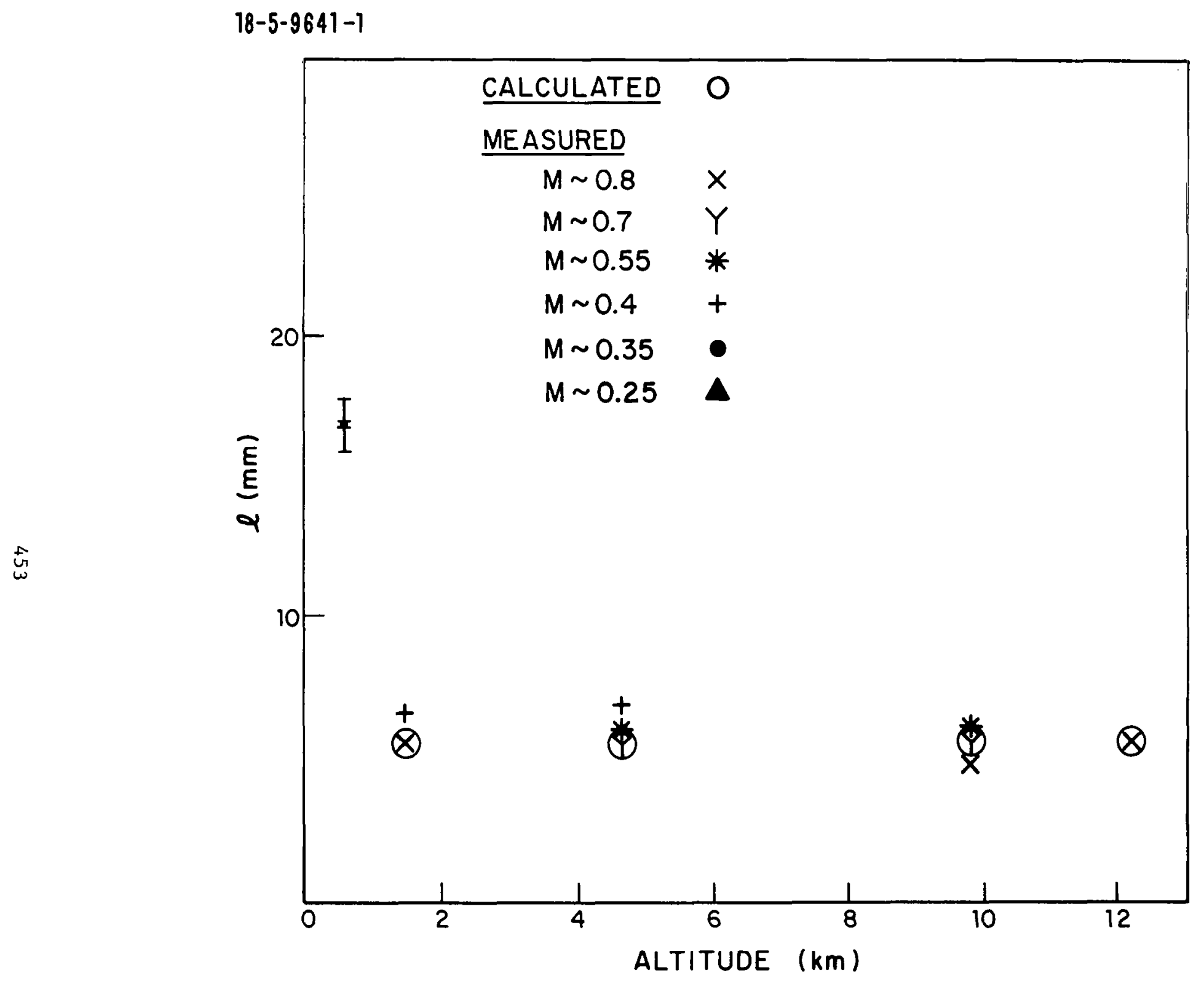

(a) LEAR JET.

FIG, 15. FLIGIT BOURDIVY LIYE? \& MITA. 
$18-5-9642-1$

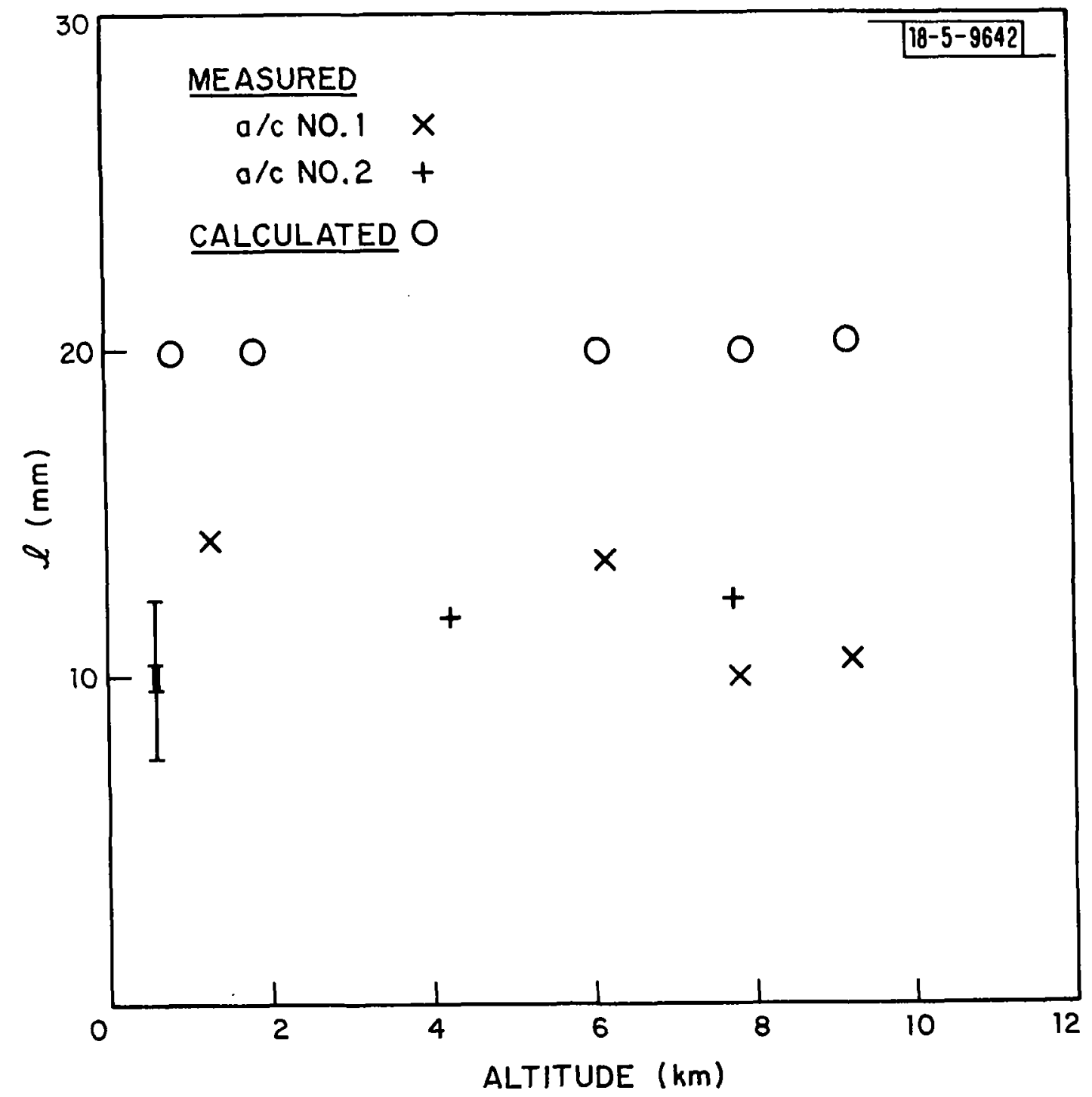

(b) KC-135 PROPAGATION EXPERIMENTS.

FIG. 15 FLIGHT BOVIDTIYY LAYER \& DATA. 


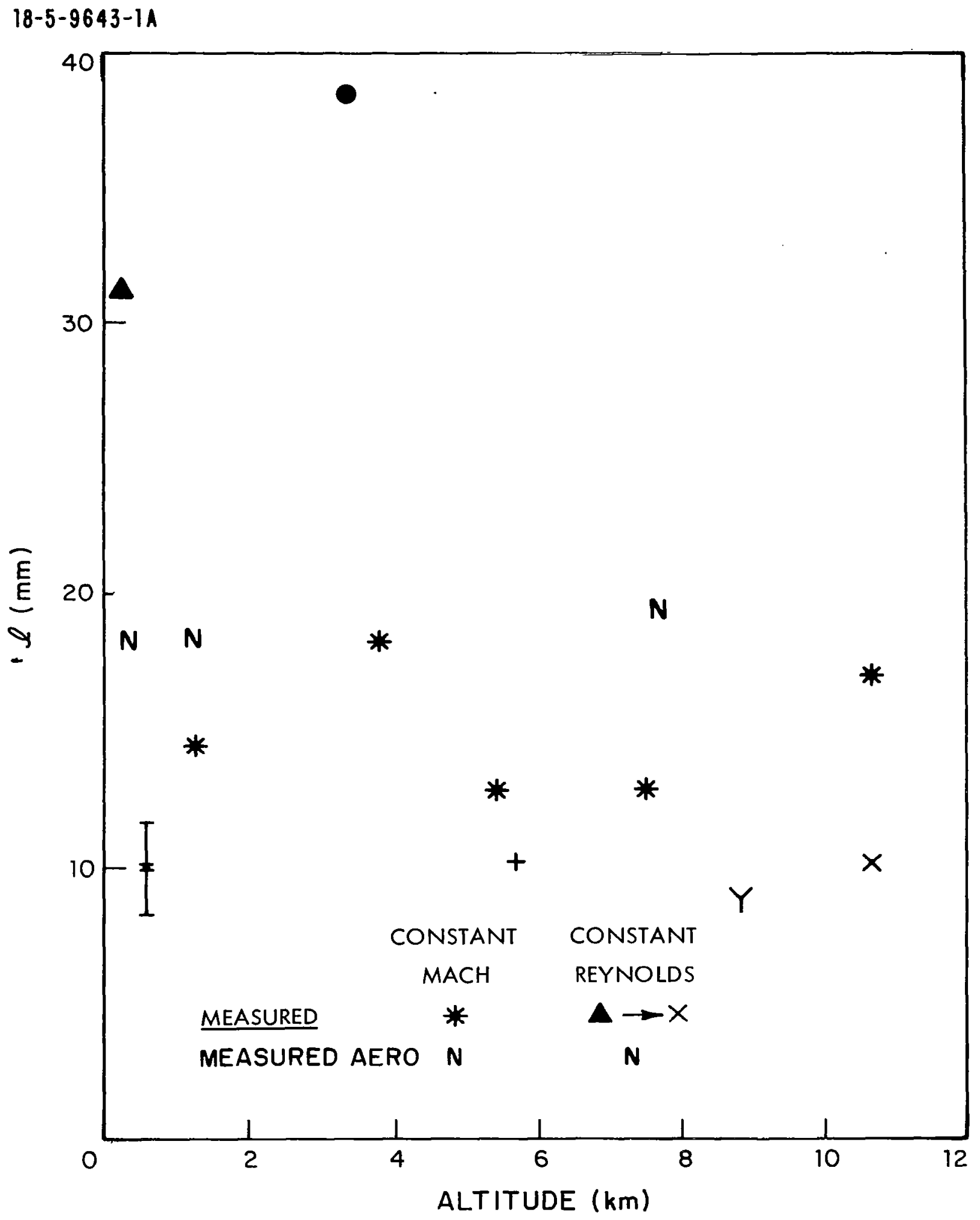

(c) KC-135 AIRFOIL EXPERIMENTS.

FIG. 15 FLIGHT BCUNDINY LAYE? \& DATA. 
$18-5-9644-1$

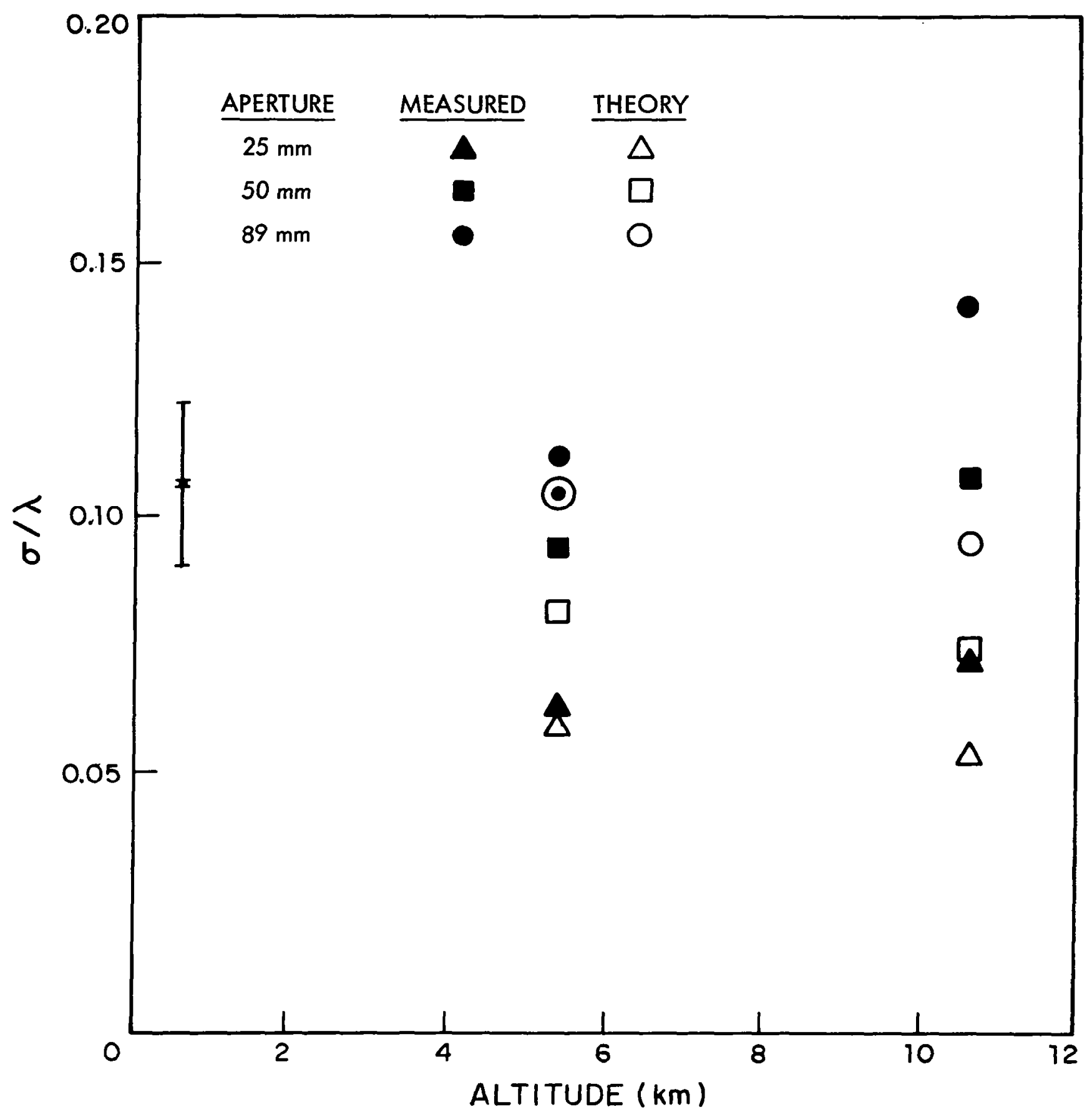

FIG, 16 o APERTURE SCALING. 
$18-5-9645-1$

ज़

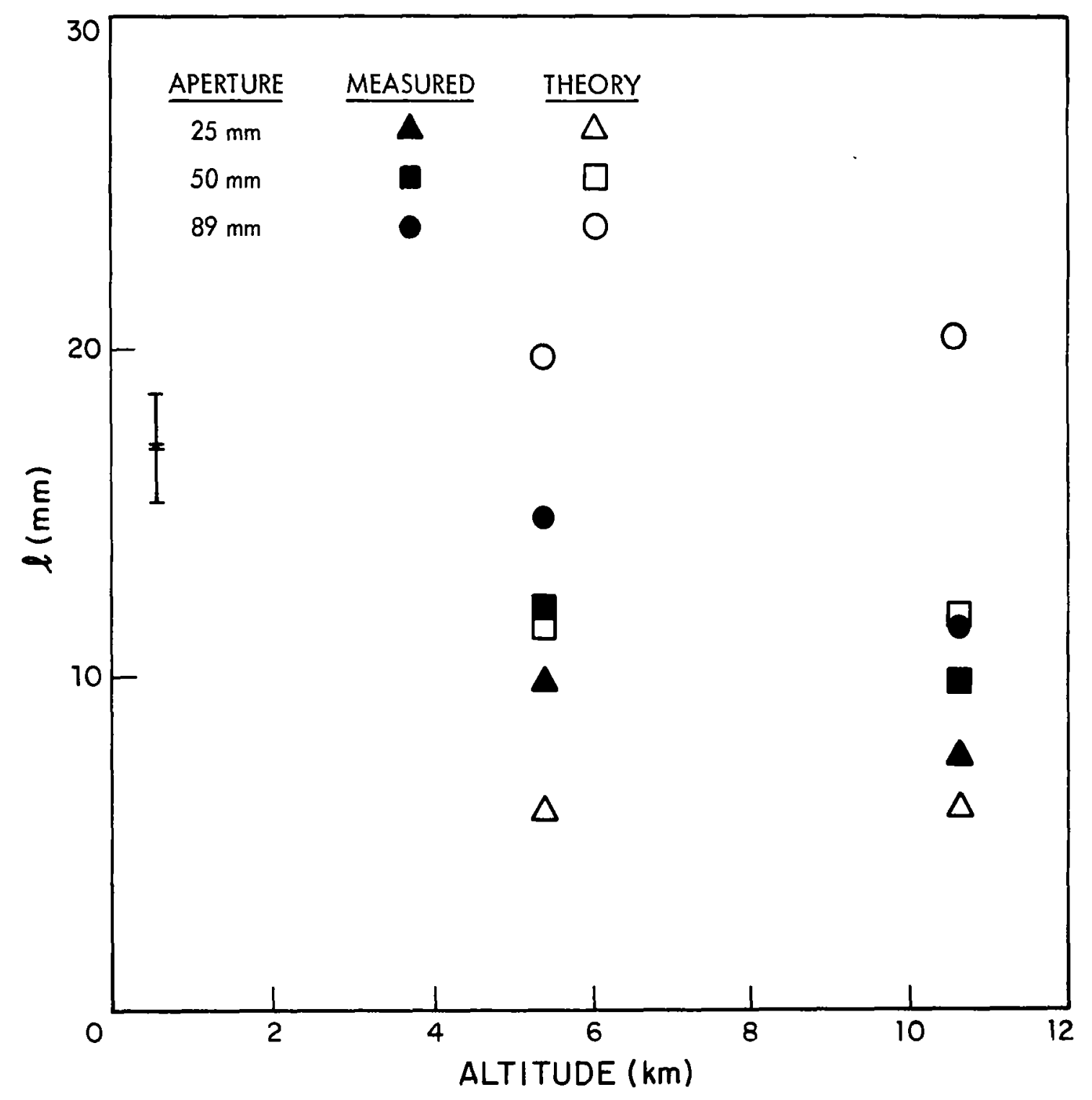

FIG 17 APERTURE SCALING. 